\title{
Convergence to a self-normalized G-Brownian motion
}

\author{
Zhengyan Lin · Li-Xin Zhang
}

Received: 25 October 2016 / Accepted: 5 January 2017 / Published online: 01 March 2017 (C) The Author(s). 2017 Open Access This article is distributed under the terms of the Creative Commons Attribution 4.0 International License (http://creativecommons.org/licenses/by/4.0/), which permits unrestricted use, distribution, and reproduction in any medium, provided you give appropriate credit to the original author(s) and the source, provide a link to the Creative Commons license, and indicate if changes were made.

\begin{abstract}
G-Brownian motion has a very rich and interesting new structure that nontrivially generalizes the classical Brownian motion. Its quadratic variation process is also a continuous process with independent and stationary increments. We prove a self-normalized functional central limit theorem for independent and identically distributed random variables under the sub-linear expectation with the limit process being a G-Brownian motion self-normalized by its quadratic variation. To prove the self-normalized central limit theorem, we also establish a new Donsker's invariance principle with the limit process being a generalized G-Brownian motion.
\end{abstract}

Keywords Sub-linear expectation · G-Brownian motion · Central limit theorem · Invariance principle $\cdot$ Self-normalization

AMS 2010 subject classifications $60 \mathrm{~F} 15 \cdot 60 \mathrm{~F} 05 \cdot 60 \mathrm{H} 10 \cdot 60 \mathrm{G} 48$

\section{Introduction}

Let $\left\{X_{n} ; n \geq 1\right\}$ be a sequence of independent and identically distributed random variables on a probability space $(\Omega, \mathscr{F}, P)$. Set $S_{n}=\sum_{j=1}^{n} X_{j}$. Suppose $E X_{1}=0$ and $E X_{1}^{2}=\sigma^{2}>0$. The well-known central limit theorem says that

$$
\frac{S_{n}}{\sqrt{n}} \stackrel{d}{\rightarrow} N\left(0, \sigma^{2}\right)
$$

Z. Lin $\cdot$ L-X. Zhang $(\bowtie)$

School of Mathematical Sciences, Zhejiang University, Hangzhou 310027 China

e-mail: stazlx@zju.edu.cn 
or, equivalently, for any bounded continuous function $\psi(x)$,

$$
E\left[\psi\left(\frac{S_{n}}{\sqrt{n}}\right)\right] \rightarrow E[\psi(\xi)],
$$

where $\xi \sim N\left(0, \sigma^{2}\right)$ is a normal random variable. If the normalization factor $\sqrt{n}$ is replaced by $\sqrt{V_{n}}$, where $V_{n}=\sum_{j=1}^{n} X_{j}^{2}$, then

$$
\frac{S_{n}}{\sqrt{V_{n}}} \stackrel{d}{\rightarrow} N(0,1) .
$$

Giné et al. (1997) proved that (3) holds if and only if $E X_{1}=0$ and

$$
\lim _{x \rightarrow \infty} \frac{x^{2} P\left(\left|X_{1}\right| \geq x\right)}{E X_{1}^{2} I\left\{\left|X_{1}\right| \leq x\right\}}=0 .
$$

The result (3) is refered to as the self-normalized central limit theorem. The purpose of this paper is to establish the self-normalized central limit theorem under the sub-linear expectation.

The sub-linear expectation, or also called G-expectation, is a nonlinear expectation generalizing the notions of backward stochastic differential equations, gexpectations, and provides a flexible framework to model non-additive probability problems and the volatility uncertainty in finance. Peng $(2006,2008 \mathrm{a}, \mathrm{b})$ introduced a general framework of the sub-linear expectation of random variables and the notions of the G-normal random variable, G-Brownian motion, independent and identically distributed random variables, etc., under the sub-linear expectation. The construction of sub-linear expectations on the space of continuous paths and discrete-time paths can also be founded in Yan et al. (2012) and Nutz and van Handel (2013). For basic properties of the sub-linear expectation, one can refer to Peng (2008b, 2009, 2010a etc.). For stochastic calculus and stochastic differential equations with respect to a G-Brownian motion, one can refer to Li and Peng (2011), Hu et al. (2014a, b), etc., and a book by Peng (2010a).

The central limit theorem under the sub-linear expectation was first established by Peng (2008b). It says that (2) remains true when the expectation $E$ is replaced by a sub-linear expectation $\hat{\mathbb{E}}$ if $\left\{X_{n} ; n \geq 1\right\}$ are independent and identically distributed under $\hat{\mathbb{E}}$, i.e.,

$$
\frac{S_{n}}{\sqrt{n}} \stackrel{d}{\rightarrow} \xi \text { under } \hat{\mathbb{E}},
$$

where $\xi$ is a G-normal random variable.

In the classical case, when $\mathrm{E}\left[X_{1}^{2}\right]$ is finite, (3) follows from the cental limit theorem (1) directly by Slutsky's lemma and the fact that

$$
\frac{V_{n}}{n} \stackrel{P}{\rightarrow} \sigma^{2}
$$

The latter is due to the law of large numbers. Under the framework of the sublinear expectation, $\frac{V_{n}}{n}$ no longer converges to a constant. The self-normalized central 
limit theorem cannot follow from the central limit theorem (5) directly. In this paper, we will prove that

$$
\frac{S_{n}}{\sqrt{V_{n}}} \stackrel{d}{\rightarrow} \frac{W_{1}}{\sqrt{\langle W\rangle_{1}}} \text { under } \hat{\mathbb{E}},
$$

where $W_{t}$ is a G-Brownian motion and $\langle W\rangle_{t}$ is its quadratic variation process. A very interesting phenomenon of G-Brownian motion is that its quadratic variation process is also a continuous process with independent and stationary increments, and thus can still be regarded as a Brownian motion. When the sub-linear expectation $\hat{\mathbb{E}}$ reduces to a linear one, $W_{t}$ is the classical Brownian motion with $W_{1} \sim N\left(0, \sigma^{2}\right)$ and $\langle W\rangle_{t}=t \sigma^{2}$, and then (6) is just (3). Our main results on the self-normalized central limit theorem will be given in Section "Main results", where the process of the self-normalized partial sums $S_{[n t]} / \sqrt{V_{n}}$ is proved to converge to a self-normalized G-Brownian motion $W_{t} / \sqrt{\langle W\rangle_{1}}$. We also consider the case in which the second moments of $X_{i}$ 's are infinite and obtain the self-normalized central limit theorem under a condition similar to (4). In the next section, we state basic settings in a sub-linear expectation space, including capacity, independence, identical distribution, G-Brownian motion, etc. One can skip this section if these concepts are familiar. To prove the self-normalized central limit theorem, we establish a new Donsker's invariance principle in Section "Invariance principle" with the limit process being a generalized G-Brownian motion. The proof is given in the last section.

\section{Basic settings}

We use the framework and notations of Peng (2008b). Let $(\Omega, \mathcal{F})$ be a given measurable space and let $\mathscr{H}$ be a linear space of real functions defined on $(\Omega, \mathcal{F})$ such that if $X_{1}, \ldots, X_{n} \in \mathscr{H}$, then $\varphi\left(X_{1}, \ldots, X_{n}\right) \in \mathscr{H}$ for each $\varphi \in C_{b}\left(\mathbb{R}^{n}\right) \cup C_{l, L i p}\left(\mathbb{R}^{n}\right)$, where $C_{b}\left(\mathbb{R}^{n}\right)$ denotes the space of all bounded continuous functions and $C_{l, L i p}\left(\mathbb{R}^{n}\right)$ denotes the linear space of (local Lipschitz) functions $\varphi$ satisfying

$$
\begin{gathered}
|\varphi(\boldsymbol{x})-\varphi(\boldsymbol{y})| \leq C\left(1+|\boldsymbol{x}|^{m}+|\boldsymbol{y}|^{m}\right)|\boldsymbol{x}-\boldsymbol{y}|, \quad \forall \boldsymbol{x}, \boldsymbol{y} \in \mathbb{R}^{n}, \\
\text { for some } C>0, m \in \mathbb{N} \text { depending on } \varphi .
\end{gathered}
$$

$\mathscr{H}$ is considered as a space of "random variables." In this case, we denote $X \in \mathscr{H}$. Further, we let $C_{b, \text { Lip }}\left(\mathbb{R}^{n}\right)$ denote the space of all bounded and Lipschitz functions on $\mathbb{R}^{n}$.

\section{Sub-linear expectation and capacity}

Definition 1 A sub-linear expectation $\hat{\mathbb{E}}$ on $\mathscr{H}$ is a function $\hat{\mathbb{E}}: \mathscr{H} \rightarrow \overline{\mathbb{R}}$ satisfying the following properties: for all $X, Y \in \mathscr{H}$, we have

(a) Monotonicity: If $X \geq Y$ then $\hat{\mathbb{E}}[X] \geq \hat{\mathbb{E}}[Y]$;

(b) Constant preserving: $\hat{\mathbb{E}}[c]=c$;

(c) Sub-additivity: $\hat{\mathbb{E}}[X+Y] \leq \hat{\mathbb{E}}[X]+\hat{\mathbb{E}}[Y]$ whenever $\hat{\mathbb{E}}[X]+\hat{\mathbb{E}}[Y]$ is not of the form $+\infty-\infty$ or $-\infty+\infty$;

(d) Positive homogeneity: $\hat{\mathbb{E}}[\lambda X]=\lambda \hat{\mathbb{E}}[X], \lambda \geq 0$. 
Here $\overline{\mathbb{R}}=[-\infty, \infty]$. The triple $(\Omega, \mathscr{H}, \hat{\mathbb{E}})$ is called a sub-linear expectation space. Given a sub-linear expectation $\hat{\mathbb{E}}$, let us denote the conjugate expectation $\widehat{\mathcal{E}}$ of $\mathbb{E}$ by

$$
\widehat{\mathcal{E}}[X]:=-\hat{\mathbb{E}}[-X], \quad \forall X \in \mathscr{H} .
$$

Next, we introduce the capacities corresponding to the sub-linear expectations. Let $\mathcal{G} \subset \mathcal{F}$. A function $V: \mathcal{G} \rightarrow[0,1]$ is called a capacity if

$$
V(\emptyset)=0, V(\Omega)=1, \text { and } V(A) \leq V(B) \forall A \subset B, A, B \in \mathcal{G} .
$$

It is called sub-additive if $V(A \cup B) \leq V(A)+V(B)$ for all $A, B \in \mathcal{G}$ with $A \cup B \in \mathcal{G}$.

Let $(\Omega, \mathscr{H}, \hat{\mathbb{E}})$ be a sub-linear space and $\widehat{\mathcal{E}}$ be the conjugate expectation of $\hat{\mathbb{E}}$. We introduce the pair $(\mathbb{V}, \mathcal{V})$ of capacities by setting

$$
\mathbb{V}(A):=\inf \left\{\hat{\mathbb{E}}[\xi]: I_{A} \leq \xi, \xi \in \mathscr{H}\right\}, \quad \mathcal{V}(A):=1-\mathbb{V}\left(A^{c}\right), \quad \forall A \in \mathcal{F},
$$

where $A^{c}$ is the complement set of $A$. Then, $\mathbb{V}$ is sub-additive and

$$
\begin{gathered}
\mathbb{V}(A)=\hat{\mathbb{E}}\left[I_{A}\right], \quad \mathcal{V}(A)=\widehat{\mathcal{E}}\left[I_{A}\right], \quad \text { if } I_{A} \in \mathscr{H} \\
\hat{\mathbb{E}}[f] \leq \mathbb{V}(A) \leq \hat{\mathbb{E}}[g], \quad \widehat{\mathcal{E}}[f] \leq \mathcal{V}(A) \leq \widehat{\mathcal{E}}[g], \quad \text { if } f \leq I_{A} \leq g, f, g \in \mathscr{H} .
\end{gathered}
$$

Further, we define an extension of $\hat{\mathbb{E}}^{*}$ of $\hat{\mathbb{E}}$ by

$$
\hat{\mathbb{E}}^{*}[X]=\inf \{\hat{\mathbb{E}}[Y]: X \leq Y, Y \in \mathscr{H}\}, \quad \forall X: \Omega \rightarrow \mathbb{R},
$$

where $\inf \emptyset=+\infty$. Then,

$$
\begin{gathered}
\hat{\mathbb{E}}^{*}[X]=\hat{\mathbb{E}}[X] \text { if } X \in \mathscr{H}, \quad \mathbb{V}(A)=\hat{\mathbb{E}}^{*}\left[I_{A}\right], \\
\hat{\mathbb{E}}[f] \leq \hat{\mathbb{E}}^{*}[X] \leq \hat{\mathbb{E}}[g] \text { if } f \leq X \leq g, f, g \in \mathscr{H} .
\end{gathered}
$$

\section{Independence and distribution}

Definition 2 (Peng $(2006,2008 b)$ )

(i) (Identical distribution) Let $\boldsymbol{X}_{1}$ and $\boldsymbol{X}_{2}$ be two n-dimensional random vectors defined, respectively, in sub-linear expectation spaces $\left(\Omega_{1}, \mathscr{H}_{1}, \hat{\mathbb{E}}_{1}\right)$ and $\left(\Omega_{2}, \mathscr{H}_{2}, \hat{\mathbb{E}}_{2}\right)$. They are called identically distributed, denoted by $\boldsymbol{X}_{1} \stackrel{d}{=}$ $\boldsymbol{X}_{2}$ if

$$
\hat{\mathbb{E}}_{1}\left[\varphi\left(\boldsymbol{X}_{1}\right)\right]=\hat{\mathbb{E}}_{2}\left[\varphi\left(\boldsymbol{X}_{2}\right)\right], \quad \forall \varphi \in C_{l, L i p}\left(\mathbb{R}^{n}\right),
$$

whenever the sub-expectations are finite. A sequence $\left\{X_{n} ; n \geq 1\right\}$ of random variables is said to be identically distributed if $X_{i} \stackrel{d}{=} X_{1}$ for each $i \geq 1$. 
(ii) (Independence) In a sub-linear expectation space $(\Omega, \mathscr{H}, \hat{\mathbb{E}})$, a random vector $\boldsymbol{Y}=\left(Y_{1}, \ldots, Y_{n}\right), Y_{i} \in \mathscr{H}$ is said to be independent to another random vector $\boldsymbol{X}=\left(X_{1}, \ldots, X_{m}\right), X_{i} \in \mathscr{H}$ under $\hat{\mathbb{E}}$ if for each test function $\varphi \in C_{l, \text { Lip }}\left(\mathbb{R}^{m} \times \mathbb{R}^{n}\right)$ we have

$$
\hat{\mathbb{E}}[\varphi(\boldsymbol{X}, \boldsymbol{Y})]=\hat{\mathbb{E}}\left[\left.\hat{\mathbb{E}}[\varphi(\boldsymbol{x}, \boldsymbol{Y})]\right|_{\boldsymbol{x}=\boldsymbol{X}}\right],
$$

whenever $\bar{\varphi}(\boldsymbol{x}):=\hat{\mathbb{E}}[|\varphi(\boldsymbol{x}, \boldsymbol{Y})|]<\infty$ for all $\boldsymbol{x}$ and $\hat{\mathbb{E}}[|\bar{\varphi}(\boldsymbol{X})|]<\infty$.

(iii) (IID random variables) A sequence of random variables $\left\{X_{n} ; n \geq 1\right\}$ is said to be independent and identically distributed (IID), if $X_{i} \stackrel{d}{=} X_{1}$ and $X_{i+1}$ is independent to $\left(X_{1}, \ldots, X_{i}\right)$ for each $i \geq 1$.

\section{G-normal distribution, G-Brownian motion and its quadratic variation}

Let $0<\underline{\sigma} \leq \bar{\sigma}<\infty$ and $G(\alpha)=\frac{1}{2}\left(\bar{\sigma}^{2} \alpha^{+}-\underline{\sigma}^{2} \alpha^{-}\right)$. $X$ is called a normal $N\left(0,\left[\underline{\sigma}^{2}, \bar{\sigma}^{2}\right]\right)$ distributed random variable (written as $\left.X \sim N\left(0,\left[\underline{\sigma}^{2}, \bar{\sigma}^{2}\right]\right)\right)$ under $\hat{\mathbb{E}}$, if for any bounded Lipschitz function $\varphi$, the function $u(x, t)=\hat{\mathbb{E}}[\varphi(x+\sqrt{t} X)]$ $(x \in \mathbb{R}, t \geq 0$ ) is the unique viscosity solution of the following heat equation:

$$
\partial_{t} u-G\left(\partial_{x x}^{2} u\right)=0, \quad u(0, x)=\varphi(x) .
$$

Let $C[0,1]$ be a function space of continuous functions on $[0,1]$ equipped with the supremum norm $\|x\|=\sup |x(t)|$ and $C_{b}(C[0,1])$ is the set of bounded continuous functions $h(x): C[0,1] \rightarrow \mathbb{R}$. The modulus of the continuity of an element $x \in C[0,1]$ is defined by

$$
\omega_{\delta}(x)=\sup _{|t-s|<\delta}|x(t)-x(s)| .
$$

It is showed that there is a sub-linear expectation space $(\widetilde{\Omega}, \widetilde{\mathscr{H}}, \widetilde{\mathbb{E}})$ with $\widetilde{\Omega}=$ $C[0,1]$ and $C_{b}(C[0,1]) \subset \widetilde{\mathscr{H}}$ such that $(\widetilde{\mathscr{H}}, \widetilde{\mathbb{E}}[\|\cdot\|])$ is a Banach space, and the canonical process $W(t)(\omega)=\omega_{t}(\omega \in \widetilde{\Omega})$ is a G-Brownian motion with $W(1) \sim$ $N\left(0,\left[\underline{\sigma}^{2}, \bar{\sigma}^{2}\right]\right)$ under $\mathbb{E}$, i.e., for all $0 \leq t_{1}<\ldots<t_{n} \leq 1, \varphi \in C_{l, l i p}\left(\mathbb{R}^{n}\right)$,

$$
\widetilde{\mathbb{E}}\left[\varphi\left(W\left(t_{1}\right), \ldots, W\left(t_{n-1}\right), W\left(t_{n}\right)-W\left(t_{n-1}\right)\right)\right]=\widetilde{\mathbb{E}}\left[\psi\left(W\left(t_{1}\right), \ldots, W\left(t_{n-1}\right)\right)\right],
$$

where $\left.\psi\left(x_{1}, \ldots, x_{n-1}\right)\right)=\widetilde{\mathbb{E}}\left[\varphi\left(x_{1}, \ldots, x_{n-1}, \sqrt{t_{n}-t_{n-1}} W(1)\right)\right]$ (cf. Peng (2006, 2008a, 2010a), Denis et al. (2011)).

The quadratic variation process of a G-Brownian motion $W$ is defined by

$$
\langle W\rangle_{t}=\lim _{\left\|\Pi_{t}^{N}\right\| \rightarrow 0} \sum_{j=1}^{N-1}\left(W\left(t_{j}^{N}\right)-W\left(t_{j-1}^{N}\right)\right)^{2}=W^{2}(t)-2 \int_{0}^{t} W(t) d W(t),
$$


where $\Pi_{t}^{N}=\left\{t_{0}^{N}, t_{1}^{N}, \ldots, t_{N}^{n}\right\}$ is a partition of $[0, t]$ and $\left\|\Pi_{t}^{N}\right\|=\max _{j}\left|t_{j}^{N}-t_{j-1}^{N}\right|$, and the limit is taken in $L_{2}$, i.e.,

$$
\lim _{\left\|\Pi_{t}^{N}\right\| \rightarrow 0} \widetilde{\mathbb{E}}\left[\left(\sum_{j=1}^{N-1}\left(W\left(t_{j}^{N}\right)-W\left(t_{j-1}^{N}\right)\right)^{2}-\langle W\rangle_{t}\right)^{2}\right]=0 .
$$

The quadratic variation process $\langle W\rangle_{t}$ is also a continuous process with independent and stationary increments. For the properties and the distribution of the quadratic variation process, one can refer to a book by Peng (2010a).

Denis et al. (2011) showed the following representation of the G-Brownian motion (cf. Theorem 52).

Lemma 1 Let $(\Omega, \mathscr{F}, P)$ be a probability measure space and $\{B(t)\}_{t \geq 0}$ is a $P$-Brownian motion. Then, for all bounded continuous functions $\varphi: C_{b}[0,1] \rightarrow \mathbb{R}$,

$$
\widetilde{\mathbb{E}}[\varphi(W(\cdot))]=\sup _{\theta \in \Theta} \mathrm{E}_{P}\left[\varphi\left(W_{\theta}(\cdot)\right)\right], \quad W_{\theta}(t)=\int_{0}^{t} \theta(s) d B(s),
$$

where

$$
\begin{gathered}
\Theta=\left\{\theta: \theta(t) \text { is an } \mathscr{F}_{t} \text {-adapted process such that } \sigma \leq \theta(t) \leq \bar{\sigma}\right\}, \\
\mathscr{F}_{t}=\sigma\{B(s): 0 \leq s \leq t\} \vee \mathscr{N}, \mathscr{N} \text { is the collection ofP-null subsets. }
\end{gathered}
$$

For the reminder of this paper, the sequences $\left\{X_{n} ; n \geq 1\right\},\left\{Y_{n} ; n \geq 1\right\}$, etc., of the random variables are considered in $(\Omega, \mathscr{H}, \hat{\mathbb{E}})$. Without specification, we suppose that $\left\{X_{n} ; n \geq 1\right\}$ is a sequence of independent and identically distributed random variables in $(\Omega, \mathscr{H}, \hat{\mathbb{E}})$ with $\hat{\mathbb{E}}\left[X_{1}\right]=\widehat{\mathcal{E}}\left[X_{1}\right]=0, \hat{\mathbb{E}}\left[X_{1}^{2}\right]=\bar{\sigma}^{2}$, and $\widehat{\mathcal{E}}\left[X_{1}^{2}\right]=\underline{\sigma}^{2}$. Denote $S_{0}^{X}=0, S_{n}^{X}=\sum_{k=1}^{n} X_{k}, V_{0}=0, V_{n}=\sum_{k=1}^{n} X_{k}^{2}$. And suppose that $(\widetilde{\Omega}, \widetilde{\mathscr{H}}, \widetilde{\mathbb{E}})$ is a sub-linear expectation space which is rich enough such that there is a G-Brownian motion $W(t)$ with $W(1) \sim N\left(0,\left[\frac{\sigma^{2}}{\widetilde{\sigma}}, \bar{\sigma}^{2}\right]\right)$. We denote a pair of capacities corresponding to the sub-linear expectation $\widetilde{\mathbb{E}}$ by $(\widetilde{\mathbb{V}}, \widetilde{\mathcal{V}})$, and the extension of $\widetilde{\mathbb{E}}$ by $\widetilde{\mathbb{E}}^{*}$.

\section{Main results}

We consider the convergence of the process $S_{[n t]}^{X}$. Because it is not in $C[0,1]$, it needs to be modified. Define the $C[0,1]$-valued random variable $\widetilde{S}_{n}^{X}(\cdot)$ by setting

$$
\tilde{S}_{n}^{X}(t)=\left\{\begin{array}{c}
\sum_{j=1}^{k} X_{j}, \text { if } t=k / n(k=0,1, \ldots, n) ; \\
\text { extended by linear interpolation in each interval } \\
{\left[[k-1] n^{-1}, k n^{-1}\right] .}
\end{array}\right.
$$

Then, $\widetilde{S}_{n}^{X}(t)=S_{[n t]}^{X}+(n t-[n t]) X_{[n t]+1}$. Here $[n t]$ is the largest integer less than or equal to $n t$. Zhang (2015) obtained the functional central limit theorem as follows. 
Theorem 1 Suppose $\hat{\mathbb{E}}\left[\left(X_{1}^{2}-b\right)^{+}\right] \rightarrow 0$ as $b \rightarrow \infty$. Then, for all bounded continuous functions $\varphi: C[0,1] \rightarrow \mathbb{R}$,

$$
\hat{\mathbb{E}}\left[\varphi\left(\frac{\widetilde{S}_{n}^{X}(\cdot)}{\sqrt{n}}\right)\right] \rightarrow \widetilde{\mathbb{E}}[\varphi(W(\cdot))]
$$

Replacing the normalization factor $\sqrt{n}$ by $\sqrt{V_{n}}$, we obtain the self-normalized process of partial sums:

$$
W_{n}(t)=\frac{\tilde{S}_{n}^{X}(t)}{\sqrt{V_{n}}},
$$

where $\frac{0}{0}$ is defined to be 0 . Our main result is the following self-normalized functional central limit theorem (FCLT).

Theorem 2 Suppose $\hat{\mathbb{E}}\left[\left(X_{1}^{2}-b\right)^{+}\right] \rightarrow 0$ as $b \rightarrow \infty$. Then, for all bounded continuous functions $\varphi: C[0,1] \rightarrow \mathbb{R}$,

$$
\hat{\mathbb{E}}^{*}\left[\varphi\left(W_{n}(\cdot)\right)\right] \rightarrow \widetilde{\mathbb{E}}\left[\varphi\left(\frac{W(\cdot)}{\sqrt{\langle W\rangle_{1}}}\right)\right] .
$$

In particular, for all bounded continuous functions $\varphi: \mathbb{R} \rightarrow \mathbb{R}$,

$$
\begin{aligned}
\hat{\mathbb{E}}^{*}\left[\varphi\left(\frac{S_{n}^{X}}{\sqrt{V_{n}}}\right)\right] \rightarrow & \widetilde{\mathbb{E}}\left[\varphi\left(\frac{W(1)}{\sqrt{\langle W\rangle_{1}}}\right)\right] \\
& =\sup _{\theta \in \Theta} E_{P}\left[\varphi\left(\frac{\int_{0}^{1} \theta(s) d B(s)}{\sqrt{\int_{0}^{1} \theta^{2}(s) d s}}\right)\right] .
\end{aligned}
$$

Remark 1 It is obvious that

$$
\widetilde{\mathbb{E}}\left[\varphi\left(\frac{W(\cdot)}{\sqrt{\langle W\rangle_{1}}}\right)\right] \geq E_{P}[\varphi(B(\cdot))] .
$$

An interesting problem is how to estimate the upper bounds of the expectations on the right hand side of (10) and (11).

Further, $\frac{W(\cdot)}{\sqrt{\langle W\rangle_{1}}} \stackrel{d}{=} \frac{\bar{W}(\cdot)}{\sqrt{\langle\bar{W}\rangle_{1}}}$, where $\bar{W}(t)$ is a G-Brownian motion with $\bar{W}(1) \sim$ $N\left(0,\left[r^{-2}, 1\right]\right), r^{2}=\bar{\sigma}^{2} / \underline{\sigma}^{2}$.

For the classical self-normalized central limit theorem, Giné et al. (1997) showed that the finiteness of the second moments can be relaxed to the condition (4). Csörgo" et al. (2003) proved the self-normalized functional central limit theorem under (4). The next theorem gives a similar result under the sub-linear expectation and is an extension of Theorem 2 . 
Theorem 3 Let $\left\{X_{n} ; n \geq 1\right\}$ be a sequence of independent and identically distributed random variables in the sub-linear expectation space $(\Omega, \mathscr{H}, \hat{\mathbb{E}})$ with $\hat{\mathbb{E}}\left[X_{1}\right]=\widehat{\mathcal{E}}\left[X_{1}\right]=0$. Denote $l(x)=\hat{\mathbb{E}}\left[X_{1}^{2} \wedge x^{2}\right]$. Suppose

(II) $\lim _{x \rightarrow \infty} \frac{\hat{\mathbb{E}}\left[X_{1}^{2} \wedge x^{2}\right]}{\hat{\mathcal{E}}\left[X_{1}^{2} \wedge x^{2}\right]}=r^{2}<\infty$;

(III) $\hat{\mathbb{E}}\left[\left(\left|X_{1}\right|-c\right)^{+}\right] \rightarrow 0$ as $c \rightarrow \infty$.

Then, the conclusions of Theorem 2 remain true with $W(t)$ being a G-Brownian motion such that $W(1) \sim N\left(0,\left[r^{-2}, 1\right]\right)$.

Remark 2 Note for $c>1, l(c x)=\hat{\mathbb{E}}\left[X_{1}^{2} \wedge(c x)^{2}\right] \leq l(x)+(c x)^{2} \mathbb{V}\left(\left|X_{1}\right| \geq x\right)$. Condition (I) implies that $l(c x) / l(x) \rightarrow 1$ as $x \rightarrow \infty$, i.e., $l(x)$ is a slowly varying function. Therefore, there is a constant $C$ such that $\int_{x}^{\infty} y^{-2} l(y) d y \leq C x^{-1} l(x)$ if $x$ is large enough. So, $\int_{x}^{\infty} \mathbb{V}\left(\left|X_{1}\right| \geq y\right) d y=o\left(x^{-1} l(x)\right)$. Also, by Lemma $3.9(b)$ of Zhang (2016), condition (III) implies that $\hat{\mathbb{E}}\left[\left(\left|X_{1}\right|-x\right)^{+}\right] \leq \int_{x}^{\infty} \mathbb{V}\left(\left|X_{1}\right| \geq y\right) d y$. Hence, $\hat{\mathbb{E}}\left[\left(\left|X_{1}\right|-x\right)^{+}\right]=o\left(x^{-1} l(x)\right)$ if conditions (I) and (III) are satisfied. When $\hat{\mathbb{E}}$ is a continuous sub-linear expectation, then for any random variable $Y$ we have $\hat{\mathbb{E}}[|Y|] \leq \int_{0}^{\infty} \mathbb{V}(|Y| \geq y) d y$ by Lemma 3.9 (c) of Zhang (2016), and so the condition (III) can be removed. Here, $\hat{\mathbb{E}}$ is called continuous if, for any $0 \leq X_{n}, X \in \mathscr{H}$ with $\hat{\mathbb{E}}\left[X_{n}\right], \hat{\mathbb{E}}[X]<\infty, \hat{\mathbb{E}}\left[X_{n}\right] \nearrow \hat{\mathbb{E}}[X]$ whenever $0 \leq X_{n} \nearrow X$, and, $\hat{\mathbb{E}}\left[X_{n}\right] \searrow \hat{\mathbb{E}}[X]$ whenever $X_{n} \searrow X$.

\section{Invariance principle}

To prove Theorems 2 and 3, we will prove a new Donsker's invariance principle. Let $\left\{\left(X_{i}, Y_{i}\right) ; i \geq 1\right\}$ be a sequence of independent and identically distributed random vectors in the sub-linear expectation space $(\Omega, \mathscr{H}, \hat{\mathbb{E}})$ with $\hat{\mathbb{E}}\left[X_{1}\right]=\hat{\mathbb{E}}\left[-X_{1}\right]=0$, $\hat{\mathbb{E}}\left[X_{1}^{2}\right]=\bar{\sigma}^{2}, \widehat{\mathcal{E}}\left[X_{1}^{2}\right]=\underline{\sigma}^{2}, \hat{\mathbb{E}}\left[Y_{1}\right]=\bar{\mu}, \widehat{\mathcal{E}}\left[Y_{1}\right]=\underline{\mu}$. Denote

$$
G(p, q)=\hat{\mathbb{E}}\left[\frac{1}{2} q X_{1}^{2}+p Y_{1}\right], \quad p, q \in \mathbb{R} .
$$

Let $\xi$ be a G-normal distributed random variable, $\eta$ be a maximal distributed random variable such that the distribution of $(\xi, \eta)$ is characterized by the following parabolic partial differential equation (PDE) defined on $[0, \infty) \times \mathbb{R} \times \mathbb{R}$ :

$$
\partial_{t} u-G\left(\partial_{y} u, \partial_{x x}^{2} u\right)=0,
$$

i.e., if for any bounded Lipschitz function $\varphi(x, y): \mathbb{R}^{2} \rightarrow \mathbb{R}$, the function $u(x, y, t)=\widetilde{E}[\varphi(x+\sqrt{t} \xi, y+t \eta)](x, y \in \mathbb{R}, t \geq 0)$ is the unique viscosity solution of the PDE (13) with Cauchy condition $\left.u\right|_{t=0}=\varphi$.

Further, let $B_{t}$ and $b_{t}$ be two random processes such that the distribution of the process $(B ., b$. ) is characterized by

(i) $B_{0}=0, b_{0}=0$; 
(ii) for any $0 \leq t_{1} \leq \ldots \leq t_{k} \leq s \leq t+s,\left(B_{s+t}-B_{s}, b_{s+t}-b_{s}\right)$ is independent to $\left(B_{t_{j}}, b_{t_{j}}\right), j=1, \ldots, k$, in sense that, for any $\varphi \in C_{l, L i p}\left(\mathbb{R}^{2(k+1)}\right)$,

$$
\begin{gathered}
\widetilde{\mathbb{E}}\left[\varphi\left(\left(B_{t_{1}}, b_{t_{1}}\right), \ldots,\left(B_{t_{k}}, b_{t_{k}}\right),\left(B_{s+t}-B_{s}, b_{s+t}-b_{s}\right)\right)\right] \\
=\widetilde{\mathbb{E}}\left[\psi\left(\left(B_{t_{1}}, b_{t_{1}}\right), \ldots,\left(B_{t_{k}}, b_{t_{k}}\right)\right)\right],
\end{gathered}
$$

where

$$
\begin{array}{r}
\psi\left(\left(x_{1}, y_{1}\right), \ldots,\left(x_{k}, y_{k}\right)\right)=\widetilde{\mathbb{E}}\left[\varphi \left(\left(x_{1}, y_{1}\right), \ldots,\left(x_{k}, y_{k}\right),\right.\right. \\
\left.\left.\left(B_{s+t}-B_{s}, b_{s+t}-b_{s}\right)\right)\right] ;
\end{array}
$$

(iii) for any $t, s>0,\left(B_{s+t}-B_{s}, b_{s+t}-b_{s}\right) \stackrel{d}{\sim}\left(B_{t}, b_{t}\right)$ under $\widetilde{\mathbb{E}}$;

(iv) for any $t>0,\left(B_{t}, b_{t}\right) \stackrel{d}{\sim}\left(\sqrt{t} B_{1}, t b_{1}\right)$ under $\widetilde{\mathbb{E}}$;

(v) the distribution of $\left(B_{1}, b_{1}\right)$ is characterized by the PDE (13).

It is easily seen that $B_{t}$ is a G-Brownian motion with $B_{1} \sim N\left(0,\left[\underline{\sigma}^{2}, \bar{\sigma}^{2}\right]\right)$, and $\left(B_{t}, b_{t}\right)$ is a generalized G-Brownian motion introduced by Peng (2010a). The existence of the generalized G-Brownian motion can be found in Peng (2010a). Let

Theorem 4 Suppose $\hat{\mathbb{E}}\left[\left(X_{1}^{2}-b\right)^{+}\right] \rightarrow 0$ and $\hat{\mathbb{E}}\left[\left(\left|Y_{1}\right|-b\right)^{+}\right] \rightarrow 0$ as $b \rightarrow \infty$.

$$
\widetilde{\boldsymbol{W}}_{n}(t)=\left(\frac{\widetilde{S}_{n}^{X}(t)}{\sqrt{n}}, \frac{\tilde{S}_{n}^{Y}(t)}{n}\right) .
$$

Then, for any bounded continuous function $\varphi: C[0,1] \times C[0,1] \rightarrow \mathbb{R}$,

$$
\lim _{n \rightarrow \infty} \hat{\mathbb{E}}\left[\varphi\left(\widetilde{\boldsymbol{W}}_{n}(\cdot)\right)\right]=\widetilde{\mathbb{E}}[\varphi(B ., b .)] .
$$

Further, let $p \geq 2, q \geq 1$, and assume $\hat{\mathbb{E}}\left[\left|X_{1}\right|^{p}\right]<\infty, \hat{\mathbb{E}}\left[\left|Y_{1}\right|^{q}\right]<\infty$. Then, for any continuous function $\varphi: C[0,1] \times C[0,1] \rightarrow \mathbb{R}$ with $|\varphi(x, y)| \leq C\left(1+\|x\|^{p}+\right.$ $\left.\|y\|^{q}\right)$

$$
\lim _{n \rightarrow \infty} \hat{\mathbb{E}}^{*}\left[\varphi\left(\widetilde{\boldsymbol{W}}_{n}(\cdot)\right)\right]=\widetilde{\mathbb{E}}[\varphi(B ., b .)] .
$$

Here $\|x\|=\sup _{0 \leq t \leq 1}|x(t)|$ for $x \in C[0,1]$.

Remark 3 When $X_{k}$ and $Y_{k}$ are random vectors in $\mathbb{R}^{d}$ with $\hat{\mathbb{E}}\left[X_{k}\right]=\hat{\mathbb{E}}\left[-X_{k}\right]=0$, $\hat{\mathbb{E}}\left[\left(\left\|X_{1}\right\|^{2}-b\right)^{+}\right] \rightarrow 0$ and $\hat{\mathbb{E}}\left[\left(\left\|Y_{1}\right\|-b\right)^{+}\right] \rightarrow 0$ as $b \rightarrow \infty$. Then, the function $G$ in (12) becomes

$$
G(p, A)=\hat{\mathbb{E}}\left[\frac{1}{2}\left\langle A X_{1}, X_{1}\right\rangle+\left\langle p, Y_{1}\right\rangle\right], \quad p \in \mathbb{R}^{d}, A \in \mathbb{S}(d),
$$

where $\mathbb{S}(d)$ is the collection of all $d \times d$ symmetric matrices. The conclusion of Theorem 4 remains true with the distribution of $\left(B_{1}, b_{1}\right)$ being characterized by the following parabolic partial differential equation defined on $[0, \infty) \times \mathbb{R}^{d} \times \mathbb{R}^{d}$ :

$$
\partial_{t} u-G\left(D_{y} u, D_{x x}^{2} u\right)=0,\left.\quad u\right|_{t=0}=\varphi,
$$

where $D_{y}=\left(\partial_{y_{i}}\right)_{i=1}^{n}$ and $D_{x x}^{2}=\left(\partial_{x_{i} x_{j}}^{2}\right)_{i, j=1}^{d}$. 
Remark 4 As a conclusion of Theorem 4, we have

$$
\hat{\mathbb{E}}\left[\varphi\left(\frac{S_{n}^{X}}{\sqrt{n}}, \frac{S_{n}^{Y}}{n}\right)\right] \rightarrow \widetilde{\mathbb{E}}\left[\varphi\left(B_{1}, b_{1}\right)\right], \varphi \in C_{b}\left(\mathbb{R}^{2}\right) .
$$

This is proved by Peng (2010a) under the conditions $\hat{\mathbb{E}}\left[\left|X_{1}\right|^{2+\delta}\right]<\infty$ and $\hat{\mathbb{E}}\left[\left|Y_{1}\right|^{1+\delta}\right]<\infty$ (cf. Theorem 3.6 and Remark 3.8 therein $)$.

When $Y_{1} \equiv 0$, (15) becomes

$$
\lim _{n \rightarrow \infty} \hat{\mathbb{E}}\left[\varphi\left(\frac{\widetilde{S}_{n}^{X}(\cdot)}{\sqrt{n}}\right)\right]=\widetilde{\mathbb{E}}[\varphi(B .)], \quad \varphi \in C_{b}(C[0,1]),
$$

which is proved by Zhang (2015).

Before the proof, we need several lemmas. For random vectors $\boldsymbol{X}_{n}$ in $(\Omega, \mathscr{H}, \hat{\mathbb{E}})$ and $\boldsymbol{X}$ in $(\widetilde{\Omega}, \widetilde{\mathscr{H}}, \widetilde{\mathbb{E}})$, we write $\boldsymbol{X}_{n} \stackrel{d}{\rightarrow} \boldsymbol{X}$ if

$$
\hat{\mathbb{E}}\left[\varphi\left(\boldsymbol{X}_{n}\right)\right] \rightarrow \widetilde{\mathbb{E}}[\varphi(\boldsymbol{X})]
$$

for any bounded continuous $\varphi$. Write $\boldsymbol{X}_{n} \stackrel{\mathbb{V}}{\rightarrow} \boldsymbol{x}$ if $\mathbb{V}\left(\left\|\boldsymbol{X}_{n}-\boldsymbol{x}\right\| \geq \epsilon\right) \rightarrow 0$ for any $\epsilon>0 .\left\{\boldsymbol{X}_{n}\right\}$ is called uniformly integrable if

$$
\lim _{b \rightarrow \infty} \limsup _{n \rightarrow \infty} \hat{\mathbb{E}}\left[\left(\left\|\boldsymbol{X}_{n}\right\|-b\right)^{+}\right]=0 .
$$

The following three lemmas are obvious.

Lemma 2 If $\boldsymbol{X}_{n} \stackrel{d}{\rightarrow} \boldsymbol{X}$ and $\varphi$ is a continuous function, then $\varphi\left(\boldsymbol{X}_{n}\right) \stackrel{d}{\rightarrow} \varphi(\boldsymbol{X})$.

Lemma 3 (Slutsky's Lemma) Suppose $\boldsymbol{X}_{n} \stackrel{d}{\rightarrow} \boldsymbol{X}, \boldsymbol{Y}_{n} \stackrel{\mathbb{V}}{\rightarrow} \boldsymbol{y}, \eta_{n} \stackrel{\mathbb{V}}{\rightarrow}$ a, where $a$ is a constant and $\boldsymbol{y}$ is a constant vector, and $\widetilde{\mathbb{V}}(\|X\|>\lambda) \rightarrow 0$ as $\lambda \rightarrow \infty$. Then, $\left(\boldsymbol{X}_{n}, \boldsymbol{Y}_{n}, \eta_{n}\right) \stackrel{d}{\rightarrow}(\boldsymbol{X}, \boldsymbol{y}, a)$, and as a result, $\eta_{n} \boldsymbol{X}_{n}+\boldsymbol{Y}_{n} \stackrel{d}{\rightarrow} a \boldsymbol{X}+\boldsymbol{y}$.

Remark 5 Suppose $\boldsymbol{X}_{n} \stackrel{d}{\rightarrow} \boldsymbol{X}$. Then, $\widetilde{\mathbb{V}}(\|\boldsymbol{X}\|>\lambda) \rightarrow 0$ as $\lambda \rightarrow \infty$ is equivalent to the tightness of $\left\{X_{n} ; n \geq 1\right\}$, i.e.,

$$
\lim _{\lambda \rightarrow \infty} \limsup _{n \rightarrow \infty} \mathbb{V}\left(\left\|\boldsymbol{X}_{n}\right\|>\lambda\right)=0,
$$

because for all $\epsilon>0$, we can define a continuous function $\varphi(x)$ such that $I\{x>$ $\lambda+\epsilon\} \leq \varphi(x) \leq I\{x>\lambda]$ and so

$$
\begin{aligned}
& \widetilde{\mathbb{V}}(\|\boldsymbol{X}\|>\lambda+\epsilon) \leq \widetilde{\mathbb{E}}[\varphi(\|\boldsymbol{X}\|)]=\lim _{n \rightarrow \infty} \hat{\mathbb{E}}\left[\varphi\left(\left\|\boldsymbol{X}_{n}\right\|\right)\right] \leq \limsup _{n \rightarrow \infty} \mathbb{V}\left(\left\|\boldsymbol{X}_{n}\right\|>\lambda\right), \\
& \limsup _{n \rightarrow \infty} \mathbb{V}\left(\left\|\boldsymbol{X}_{n}\right\|>\lambda+\epsilon\right) \leq \lim _{n \rightarrow \infty} \hat{\mathbb{E}}\left[\varphi\left(\left\|\boldsymbol{X}_{n}\right\|\right)\right]=\widetilde{\mathbb{E}}[\varphi(\|\boldsymbol{X}\|)] \leq \widetilde{\mathbb{V}}(\|\boldsymbol{X}\|>\lambda) .
\end{aligned}
$$


Lemma 4 Suppose $\boldsymbol{X}_{n} \stackrel{d}{\rightarrow} \boldsymbol{X}$.

(a) If $\left\{\boldsymbol{X}_{n}\right\}$ is uniformly integrable and $\widetilde{\mathbb{E}}\left[\left((\|\boldsymbol{X}\|-b)^{+}\right] \rightarrow 0\right.$ as $b \rightarrow \infty$, then,

$$
\hat{\mathbb{E}}\left[\boldsymbol{X}_{n}\right] \rightarrow \widetilde{\mathbb{E}}[\boldsymbol{X}] .
$$

(b) If $\sup _{n} \hat{\mathbb{E}}\left[\mid \boldsymbol{X}_{n} \|^{q}<\infty\right.$ and $\widetilde{\mathbb{E}}\left[\mid \boldsymbol{X} \|^{q}<\infty\right.$ for some $q>1$, then (17) holds.

The following lemma is proved by Zhang (2015).

Lemma 5 Suppose that $\boldsymbol{X}_{n} \stackrel{d}{\rightarrow} \boldsymbol{X}, \boldsymbol{Y}_{n} \stackrel{d}{\rightarrow} \boldsymbol{Y}, \boldsymbol{Y}_{n}$ is independent to $\boldsymbol{X}_{n}$ under $\hat{\mathbb{E}}$ and $\widetilde{\mathbb{V}}(\|\boldsymbol{X}\|>\lambda) \rightarrow 0$ and $\widetilde{\mathbb{V}}(\|\boldsymbol{Y}\|>\lambda) \rightarrow 0$ as $\lambda \rightarrow \infty$. Then $\left(\boldsymbol{X}_{n}, \boldsymbol{Y}_{n}\right) \stackrel{d}{\rightarrow}(\overline{\boldsymbol{X}}, \overline{\boldsymbol{Y}})$, where $\overline{\boldsymbol{X}} \stackrel{d}{=} \boldsymbol{X}, \overline{\boldsymbol{Y}} \stackrel{d}{=} \boldsymbol{Y}$ and $\overline{\boldsymbol{Y}}$ is independent to $\overline{\boldsymbol{X}}$ under $\widetilde{\mathbb{E}}$.

The next lemma is about the Rosenthal-type inequalities due to Zhang (2016).

Lemma 6 Let $\left\{X_{1}, \ldots, X_{n}\right\}$ be a sequence of independent random variables in $(\Omega, \mathscr{H}, \hat{\mathbb{E}})$.

(a) Suppose $p \geq 2$. Then,

$$
\begin{aligned}
\hat{\mathbb{E}}\left[\max _{k \leq n}\left|S_{k}\right|^{p}\right] \leq C_{p} & \left\{\sum_{k=1}^{n} \hat{\mathbb{E}}\left[\left|X_{k}\right|^{p}\right]+\left(\sum_{k=1}^{n} \hat{\mathbb{E}}\left[\left|X_{k}\right|^{2}\right]\right)^{p / 2}\right. \\
+ & \left.\left(\sum_{k=1}^{n}\left[\left(\widehat{\mathcal{E}}\left[X_{k}\right]\right)^{-}+\left(\hat{\mathbb{E}}\left[X_{k}\right]\right)^{+}\right]\right)^{p}\right\} .
\end{aligned}
$$

(b) Suppose $\hat{\mathbb{E}}\left[X_{k}\right] \leq 0, k=1, \ldots, n$. Then,

$$
\hat{\mathbb{E}}\left[\left|\max _{k \leq n}\left(S_{n}-S_{k}\right)\right|^{p}\right] \leq 2^{2-p} \sum_{k=1}^{n} \hat{\mathbb{E}}\left[\left|X_{k}\right|^{p}\right], \text { for } 1 \leq p \leq 2
$$

and

$$
\begin{aligned}
\hat{\mathbb{E}}\left[\left|\max _{k \leq n}\left(S_{n}-S_{k}\right)\right|^{p}\right] & \leq C_{p}\left\{\sum_{k=1}^{n} \hat{\mathbb{E}}\left[\left|X_{k}\right|^{p}\right]+\left(\sum_{k=1}^{n} \hat{\mathbb{E}}\left[\left|X_{k}\right|^{2}\right]\right)^{p / 2}\right\} \\
& \leq C_{p} n^{p / 2-1} \sum_{k=1}^{n} \hat{\mathbb{E}}\left[\left|X_{k}\right|^{p}\right], \text { for } p \geq 2 .
\end{aligned}
$$

Lemma 7 Suppose $\hat{\mathbb{E}}\left[X_{1}\right]=\hat{\mathbb{E}}\left[-X_{1}\right]=0$ and $\hat{\mathbb{E}}\left[X_{1}^{2}\right]<\infty$. Let $\bar{X}_{n, k}=$ $(-\sqrt{n}) \vee X_{k} \wedge \sqrt{n}, \widehat{X}_{n, k}=X_{k}-\bar{X}_{n, k}, \bar{S}_{n, k}^{X}=\sum_{j=1}^{k} \bar{X}_{n, j}$ and $\widehat{S}_{n, k}^{X}=\sum_{j=1}^{k} \widehat{X}_{n, j}$, $k=1, \ldots, n$. Then

$$
\hat{\mathbb{E}}\left[\max _{k \leq n}\left|\frac{\bar{S}_{n, k}^{X}}{\sqrt{n}}\right|^{q}\right] \leq C_{q}, \quad \text { for all } q \geq 2,
$$


and

$$
\lim _{n \rightarrow \infty} \hat{\mathbb{E}}\left[\max _{k \leq n}\left|\frac{\widehat{S}_{n, k}^{X}}{\sqrt{n}}\right|^{p}\right]=0
$$

whenever $\hat{\mathbb{E}}\left[\left(\left|X_{1}\right|^{p}-b\right)^{+}\right] \rightarrow 0$ as $b \rightarrow \infty$ if $p=2$, and $\hat{\mathbb{E}}\left[\left|X_{1}\right|^{p}\right]<\infty$ if $p>2$.

Proof Note $\hat{\mathbb{E}}\left[X_{1}\right]=\widehat{\mathcal{E}}\left[X_{1}\right]=0$. So, $\left|\widehat{\mathcal{E}}\left[\bar{X}_{n, 1}\right]\right|=\left|\widehat{\mathcal{E}}\left[X_{1}\right]-\widehat{\mathcal{E}}\left[\bar{X}_{n, 1}\right]\right| \leq \hat{\mathbb{E}}\left|\widehat{X}{ }_{n, 1}\right| \leq$ $\hat{\mathbb{E}}\left[\left(\left|X_{1}\right|^{2}-n\right)^{+}\right] n^{-1 / 2}$ and $\left|\hat{\mathbb{E}}\left[\bar{X}_{n, 1}\right]\right|=\left|\hat{\mathbb{E}}\left[X_{1}\right]-\hat{\mathbb{E}}\left[\bar{X}_{n, 1}\right]\right| \leq \hat{\mathbb{E}}\left|\widehat{X}_{n, 1}\right| \leq \hat{\mathbb{E}}\left[\left(\left|X_{1}\right|^{2}-\right.\right.$ $\left.n)^{+}\right] n^{-1 / 2}$. By Rosenthal's inequality (cf. (18)),

$$
\begin{aligned}
\hat{\mathbb{E}}\left[\max _{k \leq n}\left|\bar{S}_{n, k}^{X}\right|^{q}\right] \leq & C_{p}\left\{n \hat { \mathbb { E } } \left[\left|\bar{X}_{n, 1}\right|^{q}+\left(n \hat{\mathbb{E}}\left[\left|\bar{X}_{n, 1}\right|^{2}\right]\right)^{q / 2}\right.\right. \\
& \left.+\left(n\left[\left(\widehat{\mathcal{E}}\left[\bar{X}_{n, 1}\right]\right)^{-}+\left(\hat{\mathbb{E}}\left[\bar{X}_{n, 1}\right]\right)^{+}\right]\right)^{q}\right\} \\
\leq & C_{q}\left\{n n^{q / 2-1} \hat{\mathbb{E}}\left[\left|X_{1}\right|^{2}\right]+n^{q / 2}\left(\hat{\mathbb{E}}\left[X_{1}^{2}\right]\right)^{q / 2}+\left(n n^{-1 / 2} \hat{\mathbb{E}}\left[\left(X_{1}^{2}-n\right)^{+}\right]\right)^{q}\right\} \\
\leq & C_{q} n^{q / 2}\left\{\hat{\mathbb{E}}\left[\left|X_{1}\right|^{2}\right]+\left(\hat{\mathbb{E}}\left[X_{1}^{2}\right]\right)^{q}\right\}, \text { for all } q \geq 2
\end{aligned}
$$

and

$$
\begin{aligned}
\hat{\mathbb{E}}\left[\max _{k \leq n}\left|\widehat{S}_{n, k}^{X}\right|^{p}\right] \leq & C_{p}\left\{n \hat{\mathbb{E}}\left[\left|\widehat{X}_{n, 1}\right|^{p}\right]+\left(n \hat{\mathbb{E}}\left[\left|\widehat{X}_{n, 1}\right|^{2}\right]\right)^{p / 2}\right. \\
& \left.+\left(n\left[\left(\widehat{\mathcal{E}}\left[\widehat{X}_{n, 1}\right]\right)^{-}+\left(\hat{\mathbb{E}}\left[\widehat{X}_{n, 1}\right]\right)^{+}\right]\right)^{p}\right\} \\
\leq & C_{p}\left\{n \hat{\mathbb{E}}\left[\left(\left|X_{1}\right|^{p}-n^{p / 2}\right)^{+}\right]+n^{p / 2}\left(\hat{\mathbb{E}}\left[\left(X_{1}^{2}-n\right)^{+}\right]\right)^{p / 2}\right. \\
& \left.+n^{p / 2}\left(\hat{\mathbb{E}}\left[\left(X_{1}^{2}-n\right)^{+}\right]\right)^{p}\right\}, p \geq 2 .
\end{aligned}
$$

The proof is completed.

Lemma 8 (a) Suppose $p \geq 2, \hat{\mathbb{E}}\left[X_{1}\right]=\hat{\mathbb{E}}\left[-X_{1}\right]=0, \hat{\mathbb{E}}\left[\left(X_{1}^{2}-b\right)^{+}\right] \rightarrow 0$ as $b \rightarrow \infty$ and $\hat{\mathbb{E}}\left[\left|X_{1}\right|^{p}\right]<\infty$. Then,

$$
\left\{\max _{k \leq n}\left|\frac{S_{k}^{X}}{\sqrt{n}}\right|^{p}\right\}_{n=1}^{\infty} \text { is uniformly integrable and therefore is tight. }
$$

(b) Suppose $p \geq 1$, $\hat{\mathbb{E}}\left[\left(\left|Y_{1}\right|-b\right)^{+}\right] \rightarrow 0$ as $b \rightarrow \infty$, and $\hat{\mathbb{E}}\left[\left|Y_{1}\right|^{p}\right]<\infty$. Then,

$$
\left\{\max _{k \leq n}\left|\frac{S_{k}^{Y}}{n}\right|^{p}\right\}_{n=1}^{\infty} \text { is uniformly integrable and therefore is tight. }
$$


Proof (a) follows from Lemma 6. (b) is obvious by noting

$$
\begin{aligned}
& \left.\hat{\mathbb{E}}\left[\left(\frac{\max _{k \leq n}\left|S_{k}^{Y}\right|}{n}-b\right)^{+}\right)^{p}\right] \leq \hat{\mathbb{E}}\left[\left(\frac{\sum_{k=1}^{n}\left(\left|Y_{k}\right|-b\right)^{+}}{n}\right)^{p}\right] \\
\leq & C_{p}\left(\frac{\sum_{k=1}^{n} \hat{\mathbb{E}}\left[\left(\left|Y_{k}\right|-b\right)^{+}\right]}{n}\right)^{p} \\
& \quad+C_{p} \frac{\hat{\mathbb{E}}\left[\left|\left(\sum_{k=1}^{n}\left\{\left(\left|Y_{k}\right|-b\right)^{+}-\hat{\mathbb{E}}\left[\left(\left|Y_{k}\right|-b\right)^{+}\right]\right\}\right)^{+}\right|^{p}\right]}{n^{p}} \\
\leq & C_{p}\left(\hat{\mathbb{E}}\left[\left(\left|Y_{1}\right|-b\right)^{+}\right]\right)^{p}+C_{p}\left(n^{-p / 2}+n^{1-p}\right) \hat{\mathbb{E}}\left[\left(\left|Y_{1}\right|^{p}-b^{p}\right)^{+}\right]
\end{aligned}
$$

by the Rosenthal-type inequalities (19) and (20).

Lemma 9 Suppose $\hat{\mathbb{E}}\left[\left(\left|Y_{1}\right|-b\right)^{+}\right] \rightarrow 0$ as $b \rightarrow \infty$. Then, for any $\epsilon>0$,

$$
\mathbb{V}\left(\frac{S_{n}^{Y}}{n}>\hat{\mathbb{E}}\left[Y_{1}\right]+\epsilon\right) \rightarrow 0 \text { and } \mathbb{V}\left(\frac{S_{n}^{Y}}{n}<\widehat{\mathcal{E}}\left[Y_{1}\right]-\epsilon\right) \rightarrow 0
$$

Proof Let $Y_{k, b}=(-b) \vee Y_{k} \wedge b, S_{n, 1}=\sum_{k=1}^{n} Y_{k, b}$ and $S_{n, 2}=S_{n}^{Y}-S_{n, 1}$. Note $\hat{\mathbb{E}}\left[Y_{1, b}\right] \rightarrow \hat{\mathbb{E}}\left[Y_{1}\right]$ as $b \rightarrow \infty$. Suppose $\left|\hat{\mathbb{E}}\left[Y_{1, b}\right]-\hat{\mathbb{E}}\left[Y_{1}\right]\right|<\epsilon / 4$. Then, by Kolmogorov's inequality (cf. (19)),

$$
\begin{aligned}
& \mathbb{V}\left(\frac{S_{n, 1}}{n}>\hat{\mathbb{E}}\left[Y_{1}\right]+\epsilon / 2\right) \leq \mathbb{V}\left(\frac{S_{n, 1}}{n}>\hat{\mathbb{E}}\left[Y_{1, b}\right]+\epsilon / 4\right) \\
\leq & \frac{16}{n^{2} \epsilon^{2}} \hat{\mathbb{E}}\left[\left(\left(\sum_{k=1}^{n}\left(Y_{k, b}-\hat{\mathbb{E}}\left[Y_{k, b}\right]\right)\right)^{+}\right)^{2}\right] \\
\leq & \frac{32}{n^{2} \epsilon^{2}} \sum_{k=1}^{n} \hat{\mathbb{E}}\left[\left(Y_{k, b}-\hat{\mathbb{E}}\left[Y_{k, b}\right]\right)^{2}\right] \leq \frac{32(2 b)^{2}}{n \epsilon^{2}} \rightarrow 0 .
\end{aligned}
$$

Also,

$\mathbb{V}\left(\frac{S_{n, 2}}{n}>\epsilon / 2\right) \leq \frac{2}{n \epsilon} \sum_{k=1}^{n} \hat{\mathbb{E}}\left|Y_{k}-Y_{k, b}\right| \leq \frac{2}{\epsilon} \hat{\mathbb{E}}\left[\left(\left|Y_{1}\right|-b\right)^{+}\right] \rightarrow 0$ as $b \rightarrow \infty$.

It follows that

$$
\mathbb{V}\left(\frac{S_{n}^{Y}}{n}>\hat{\mathbb{E}}\left[Y_{1}\right]+\epsilon\right) \rightarrow 0 .
$$

By considering $\left\{-Y_{k}\right\}$ instead, we have

$$
\mathbb{V}\left(\frac{S_{n}^{Y}}{n}<\widehat{\mathcal{E}}\left[Y_{1}\right]-\epsilon\right)=\mathbb{V}\left(\frac{-S_{n}^{Y}}{n}>\hat{\mathbb{E}}\left[-Y_{1}\right]+\epsilon\right) \rightarrow 0
$$


Proof of Theorem 4. We first show the tightness of $\widetilde{\boldsymbol{W}}_{n}$. It is easily seen that

$$
w_{\delta}\left(\frac{\tilde{S}_{n}^{Y}(\cdot)}{n}\right) \leq 2 \delta b+\frac{\sum_{k=1}^{n}\left(\left|Y_{k}\right|-b\right)^{+}}{n} .
$$

It follows that for any $\epsilon>0$, if $\delta<\epsilon /(4 b)$, then

$$
\sup _{n} \mathbb{V}\left(w_{\delta}\left(\frac{\tilde{S}_{n}^{Y}(\cdot)}{n}\right) \geq \epsilon\right) \leq \sup _{n} \mathbb{V}\left(\sum_{k=1}^{n}\left(\left|Y_{k}\right|-b\right)^{+} \geq n \frac{\epsilon}{2}\right) \leq \frac{2}{\epsilon} \hat{\mathbb{E}}\left[\left(\left|Y_{1}\right|-b\right)^{+}\right] \text {. }
$$

Letting $\delta \rightarrow 0$ and then $b \rightarrow \infty$ yields

$$
\sup _{n} \mathbb{V}\left(w_{\delta}\left(\frac{\widetilde{S}_{n}^{Y}(\cdot)}{n}\right) \geq \epsilon\right) \rightarrow 0 \text { as } \delta \rightarrow 0 .
$$

For any $\eta>0$, we choose $\delta_{k} \downarrow 0$ such that, if

$$
A_{k}=\left\{x: \omega_{\delta_{k}}(x)<\frac{1}{k}\right\},
$$

then $\sup _{n} \mathbb{V}\left(\widetilde{S}_{n}^{Y}(\cdot) / n \in A_{k}^{c}\right) \leq \eta / 2^{k+1}$. Let $A=\{x:|x(0)| \leq a\}, K_{2}=A \bigcap_{k=1}^{\infty} A_{k}$. Then, by the Arzelá-Ascoli theorem, $K_{2} \subset C_{b}(C[0,1])$ is compact. It is obvious that $\left\{\widetilde{S}_{n}^{Y}(\cdot) / n \notin A\right\}=\emptyset$, because $\widetilde{S}_{n}^{Y}(0) / n=0$. Next, we show that

$$
\mathbb{V}\left(\tilde{S}_{n}^{Y}(\cdot) / n \in K_{2}^{c}\right) \leq \mathbb{V}\left(\tilde{S}_{n}^{Y}(\cdot) / n \in A^{c}\right)+\sum_{k=1}^{\infty} \mathbb{V}\left(\widetilde{S}_{n}^{Y}(\cdot) / n \in A_{k}^{c}\right) .
$$

Note that when $\delta<1 /(2 n)$,

$$
\omega_{\delta}\left(\tilde{S}_{n}^{Y}(\cdot) / n\right) \leq 2 n|t-s| \max _{i \leq n}\left|Y_{i}\right| / n \leq 2 \delta \max _{i \leq n}\left|Y_{i}\right| .
$$

Choose a $k_{0}$ such that $\delta_{k}<1 /(2 M k)$ for $k \geq k_{0}$. Then, on the event $E=$ $\left\{\max _{i \leq n}\left|Y_{i}\right| \leq M\right\},\left\{\widetilde{S}_{n}^{Y}(\cdot) / n \in A_{k}^{c}\right\}=\emptyset$ for $k \geq k_{0}$. So, by the (finite) sub-additivity of $\mathbb{V}$,

$$
\begin{aligned}
& \mathbb{V}\left(E \bigcap\left\{\tilde{S}_{n}^{Y}(\cdot) / n \in K^{c}\right\}\right) \\
\leq & \mathbb{V}\left(E \bigcap\left\{\tilde{S}_{n}^{Y}(\cdot) / n \in A^{c}\right\}\right)+\sum_{k=1}^{k_{0}} \mathbb{V}\left(E \bigcap\left\{\tilde{S}_{n}^{Y}(\cdot) / n \in A_{k}^{c}\right\}\right) \\
\leq & \mathbb{V}\left(\tilde{S}_{n}^{Y}(\cdot) / n \in A^{c}\right)+\sum_{k=1}^{\infty} \mathbb{V}\left(\tilde{S}_{n}^{Y}(\cdot) / n \in A_{k}^{c}\right) .
\end{aligned}
$$

On the other hand,

It follows that

$$
\mathbb{V}\left(E^{c}\right) \leq \frac{\hat{\mathbb{E}}\left[\max _{i \leq n}\left|Y_{i}\right|\right]}{M} \leq \frac{n \hat{\mathbb{E}}\left[\left|Y_{1}\right|\right]}{M} .
$$

$\mathbb{V}\left(\widetilde{S}_{n}^{Y}(\cdot) / n \in K_{2}^{c}\right) \leq \mathbb{V}\left(\widetilde{S}_{n}^{Y}(\cdot) / n \in A^{c}\right)+\sum_{k=1}^{\infty} \mathbb{V}\left(\widetilde{S}_{n}^{Y}(\cdot) / n \in A_{k}^{c}\right)+\frac{n \hat{\mathbb{E}}\left[\left|Y_{1}\right|\right]}{M}$ 
Letting $M \rightarrow \infty$ yields

$$
\begin{aligned}
\mathbb{V}\left(\widetilde{S}_{n}^{Y}(\cdot) / n \in K_{2}^{c}\right) & \leq \mathbb{V}\left(\tilde{S}_{n}^{Y}(\cdot) / n \in A^{c}\right)+\sum_{k=1}^{\infty} \mathbb{V}\left(\widetilde{S}_{n}^{Y}(\cdot) / n \in A_{k}^{c}\right) \\
& <0+\sum_{k=1}^{\infty} \frac{\eta}{2^{k+1}}<\frac{\eta}{2}
\end{aligned}
$$

We conclude that for any $\eta>0$, there exists a compact $K_{2} \subset C_{b}(C[0,1])$ such that

$$
\sup _{n} \hat{\mathbb{E}}^{*}\left[I\left\{\frac{\tilde{S}_{n}^{Y}(\cdot)}{n} \notin K_{2}\right\}\right]=\sup _{n} \mathbb{V}\left\{\frac{\widetilde{S}_{n}^{Y}(\cdot)}{n} \notin K_{2}\right\}<\eta / 2 .
$$

Next, we show that for any $\eta>0$, there exists a compact $K_{1} \subset C_{b}(C[0,1])$ such that

$$
\sup _{n} \hat{\mathbb{E}}^{*}\left[I\left\{\frac{\tilde{S}_{n}^{X}(\cdot)}{\sqrt{n}} \notin K_{1}\right\}\right]=\sup _{n} \mathbb{V}\left\{\frac{\tilde{S}_{n}^{X}(\cdot)}{\sqrt{n}} \notin K_{1}\right\}<\eta / 2 .
$$

Similar to (21), it is sufficient to show that

$$
\sup _{n} \mathbb{V}\left(w_{\delta}\left(\frac{\tilde{S}_{n}^{X}(\cdot)}{\sqrt{n}}\right) \geq \epsilon\right) \rightarrow 0 \text { as } \delta \rightarrow 0 .
$$

With the same argument of Billingsley (1968, Pages 56-59, cf. (8.12)), for large $n$,

$$
\begin{aligned}
& \mathbb{V}\left(w_{\delta}\left(\frac{\widetilde{S}_{n}^{X}(\cdot)}{\sqrt{n}}\right) \geq 3 \epsilon\right) \leq \frac{2}{\delta} \mathbb{V}\left(\max _{i \leq[n \delta]} \frac{\left|S_{i}^{X}\right|}{\sqrt{[n \delta]}} \geq \epsilon \frac{\sqrt{n}}{\sqrt{[n \delta]}}\right) \\
\leq & \frac{2}{\delta} \mathbb{V}\left(\max _{i \leq[n \delta]} \frac{\left|S_{i}^{X}\right|}{\sqrt{[n \delta]}} \geq \frac{\epsilon}{\sqrt{2 \delta}}\right) \leq \frac{4}{\epsilon^{2}} \hat{\mathbb{E}}\left[\left(\max _{i \leq[n \delta]}\left|\frac{S_{i}^{X}}{\sqrt{[n \delta]}}\right|^{2}-\frac{\epsilon^{2}}{2 \delta}\right)^{+}\right] .
\end{aligned}
$$

It follows that

$$
\lim _{\delta \rightarrow 0} \limsup _{n \rightarrow \infty} \mathbb{V}\left(w_{\delta}\left(\frac{\widetilde{S}_{n}^{X}(\cdot)}{\sqrt{n}}\right) \geq 3 \epsilon\right)=0
$$

by Lemma 8 (a), where $p=2$. On the other hand, for fixed $n$, if $\delta<1 /(2 n)$, then

$$
\omega_{\delta}\left(\widetilde{S}_{n}^{X}(\cdot) / \sqrt{n}\right) \leq 2 n|t-s| \max _{i \leq n}\left|X_{i}\right| / \sqrt{n} \leq 2 \delta \sqrt{n} \max _{i \leq n}\left|X_{i}\right| .
$$

We have

$$
\lim _{\delta \rightarrow 0} \mathbb{V}\left(w_{\delta}\left(\frac{\widetilde{S}_{n}^{X}(\cdot)}{\sqrt{n}}\right) \geq \epsilon\right)=0
$$

for each $n$. It follows that (23) holds.

Now, by combining (21) and (22) we obtain the tightness of $\widetilde{\boldsymbol{W}}_{n}$ as follows.

$$
\sup _{n} \hat{\mathbb{E}}^{*}\left[I\left\{\widetilde{\boldsymbol{W}}_{n}(\cdot) \notin K_{1} \times K_{2}\right\}\right]<\eta \text {. }
$$


Define $\hat{\mathbb{E}}_{n}$ by

$$
\hat{\mathbb{E}}_{n}[\varphi]=\hat{\mathbb{E}}\left[\varphi\left(\widetilde{\boldsymbol{W}}_{n}(\cdot)\right)\right], \quad \varphi \in C_{b}(C[0,1] \times C[0,1]) .
$$

Then, the sequence of sub-linear expectations $\left\{\hat{\mathbb{E}}_{n}\right\}_{n=1}^{\infty}$ is tight by (24). By Theorem 9 of Peng (2010b), $\left\{\hat{\mathbb{E}}_{n}\right\}_{n=1}^{\infty}$ is weakly compact, namely, for each subsequence $\left\{\hat{\mathbb{E}}_{n_{k}}\right\}_{k=1}^{\infty}, n_{k} \rightarrow \infty$, there exists a further subsequence $\left\{\hat{\mathbb{E}}_{m_{j}}\right\}_{j=1}^{\infty} \subset\left\{\hat{\mathbb{E}}_{n_{k}}\right\}_{k=1}^{\infty}$, $m_{j} \rightarrow \infty$, such that, for each $\varphi \in C_{b}(C[0,1] \times C[0,1]),\left\{\hat{\mathbb{E}}_{m_{j}}[\varphi]\right\}$ is a Cauchy sequence. Define $\mathbb{F}[\cdot]$ by

$$
\mathbb{F}[\varphi]=\lim _{j \rightarrow \infty} \hat{\mathbb{E}}_{m_{j}}[\varphi], \varphi \in C_{b}(C[0,1] \times C[0,1])
$$

Let $\bar{\Omega}=C[0,1] \times C[0,1]$, and $\left(\xi_{t}, \eta_{t}\right)$ be the canonical process $\xi_{t}(\omega)=\omega_{t}^{(1)}$, $\eta_{t}(\omega)=\omega_{t}^{(2)}\left(\omega=\left(\omega^{(1)}, \omega^{(2)}\right) \in \bar{\Omega}\right)$. Then,

$$
\hat{\mathbb{E}}\left[\varphi\left(\widetilde{\boldsymbol{W}}_{m_{j}}(\cdot)\right)\right] \rightarrow \mathbb{F}[\varphi(\xi ., \eta .)], \varphi \in C_{b}(C[0,1] \times C[0,1]) .
$$

The topological completion of $C_{b}(\bar{\Omega})$ under the Banach norm $\mathbb{F}[\|\cdot\|]$ is denoted by $L_{\mathbb{F}}(\bar{\Omega}) . \mathbb{F}[\cdot]$ can be extended uniquely to a sub-linear expectation on $L_{\mathbb{F}}(\bar{\Omega})$.

Next, it is sufficient to show that $\left(\xi_{t}, \eta_{t}\right)$ defined on the sub-linear space $\left(\bar{\Omega}, L_{\mathbb{F}}(\bar{\Omega}), \mathbb{F}\right)$ satisfies (i)-(v) and so $(\xi ., \eta$. $) \stackrel{d}{=}(B ., b$. $)$, which means that the limit distribution of any subsequence of $\widetilde{\boldsymbol{W}}_{n}(\cdot)$ is uniquely determined.

The conclusion in (i) is obvious. For (ii) and (iii), we let $0 \leq t_{1} \leq \ldots \leq t_{k} \leq s \leq$ $t+s$. By (25), for any bounded continuous function $\varphi: \mathbb{R}^{2(k+1)} \rightarrow \mathbb{R}$ we have

$$
\begin{aligned}
& \hat{\mathbb{E}}\left[\varphi\left(\widetilde{W}_{m_{j}}\left(t_{1}\right), \ldots, \widetilde{W}_{m_{j}}\left(t_{k}\right), \widetilde{W}_{m_{j}}(s+t)-\widetilde{W}_{m_{j}}(s)\right)\right] \\
\rightarrow & \mathbb{F}\left[\varphi\left(\left(\xi_{t_{1}}, \eta_{t_{1}}\right), \ldots,\left(\xi_{t_{k}}, \eta_{t_{k}}\right),\left(\xi_{s+t}-\xi_{s}, \eta_{s+t}-\eta_{s}\right)\right)\right] .
\end{aligned}
$$

Note

$$
\begin{aligned}
& \sup _{0 \leq t \leq 1} \frac{\left|\tilde{S}_{n}^{X}(t)-S_{[n t]}^{X}\right|}{\sqrt{n}} \leq \frac{\max _{k \leq n}\left|X_{k}\right|}{\sqrt{n}} \stackrel{\mathbb{V}}{\rightarrow} 0, \\
& \sup _{0 \leq t \leq 1} \frac{\left|\tilde{S}_{n}^{Y}(t)-S_{[n t]}^{Y}\right|}{n} \leq \frac{\max _{k \leq n}\left|Y_{k}\right|}{n} \stackrel{\mathbb{V}}{\rightarrow} 0 .
\end{aligned}
$$

It follows that by Lemmas 3 and 8,

$$
\begin{aligned}
\hat{\mathbb{E}}[\varphi & \left(\left(\frac{S_{\left[m_{j} t_{1}\right]}^{X}}{\sqrt{m_{j}}}, \frac{S_{\left[m_{j} t_{1}\right]}^{Y}}{m_{j}}\right), \ldots,\left(\frac{\left.S_{\left[m_{j} t_{k}\right]}^{X}, \frac{S_{\left[m_{j} t_{k}\right]}^{Y}}{m_{j}}\right),}{m_{j}}\right)\right. \\
& \left(\frac{\left.\left.\left.S_{\left[m_{j}(s+t)\right]}^{X}-S_{\left[m_{j} s\right]}^{X}, \frac{S_{\left[m_{j}(s+t)\right]}^{Y}-S_{\left[m_{j} s\right]}^{Y}}{m_{j}}\right)\right)\right]}{\sqrt{m_{j}}}, \frac{\mathbb{F}\left[\varphi\left(\left(\xi_{t_{1}}, \eta_{t_{1}}\right), \ldots,\left(\xi_{t_{k}}, \eta_{t_{k}}\right),\left(\xi_{s+t}-\xi_{s}, \eta_{s+t}-\eta_{s}\right)\right)\right] .}{}\right.
\end{aligned}
$$


In particular,

$$
\begin{aligned}
& \left(\frac{S_{\left[m_{j}(s+t)\right]-\left[m_{j} s\right]}^{X}}{\sqrt{m_{j}}}, \frac{S_{\left[m_{j}(s+t)\right]-\left[m_{j} s\right]}^{Y}}{m_{j}}\right) \stackrel{d}{=}\left(\frac{S_{\left[m_{j}(s+t)\right]}^{X}-S_{\left[m_{j} s\right]}^{X}}{\sqrt{m_{j}}}, \frac{S_{\left[m_{j}(s+t)\right]}^{Y}-S_{\left[m_{j} s\right]}^{Y}}{m_{j}}\right) \\
& \stackrel{d}{\rightarrow}\left(\xi_{s+t}-\xi_{s}, \eta_{s+t}-\eta_{s}\right) .
\end{aligned}
$$

It follows that

$$
\left(\frac{S_{\left[m_{j} t\right]}^{X}}{\sqrt{m_{j}}}, \frac{S_{\left[m_{j} t\right]}^{Y}}{m_{j}}\right) \stackrel{d}{\rightarrow}\left(\xi_{s+t}-\xi_{s}, \eta_{s+t}-\eta_{s}\right) .
$$

On the other hand,

$$
\left(\frac{S_{\left[m_{j} t\right]}^{X}}{\sqrt{m_{j}}}, \frac{S_{\left[m_{j} t\right]}^{Y}}{m_{j}}\right) \stackrel{d}{\rightarrow}\left(\xi_{t}, \eta_{t}\right)
$$

by (26). Hence,

$$
\mathbb{F}\left[\phi\left(\xi_{s+t}-\xi_{s}, \eta_{s+t}-\eta_{s}\right)\right]=\mathbb{F}\left[\phi\left(\xi_{t}, \eta_{t}\right)\right] \text { for all } \phi \in C_{b}\left(\mathbb{R}^{2}\right) .
$$

Next, we show that

$\mathbb{F}\left[\left|\xi_{s+t}-\xi_{s}\right|^{p}\right] \leq C_{p} t^{p / 2}$ and $\mathbb{F}\left[\left|\eta_{s+t}-\eta_{s}\right|^{p}\right] \leq C_{p} t^{p}$, for all $p \geq 2$ and $t, s \geq 0$.

By Lemma 9,

$$
\tilde{\mathcal{V}}\left(t \underline{\mu}-\epsilon \leq \eta_{s+t}-\eta_{s} \leq t \bar{\mu}+\epsilon\right)=1 \text { for all } \epsilon>0 .
$$

It follows that

$$
\mathbb{F}\left[\left|\eta_{s+t}-\eta_{s}\right|^{p}\right] \leq t^{p}\left|\hat{\mathbb{E}}\left[\left|Y_{1}\right|\right]\right|^{p}
$$

For considering $\xi_{s+t}-\xi_{s}$, we let $\bar{S}_{n, k}^{X}$ and $\widehat{S}_{n, k}^{X}$ be defined as in Lemma 7. Then, $S_{k}^{X}=\bar{S}_{n, k}^{X}+\widehat{S}_{n, k}^{X}$. By (27) and Lemmas 7 and 3,

$$
\frac{\bar{S}_{\left[m_{j} t\right],\left[m_{j} t\right]}^{X}}{\sqrt{m_{j}}} \stackrel{d}{\rightarrow} \xi_{s+t}-\xi_{s} \text { and } \hat{\mathbb{E}}\left[\left|\frac{\bar{S}_{\left[m_{j} t\right],\left[m_{j} t\right]}^{X}}{\sqrt{m_{j}}}\right|^{p}\right] \leq C_{p} t^{p / 2}, p \geq 2 .
$$

It follows that

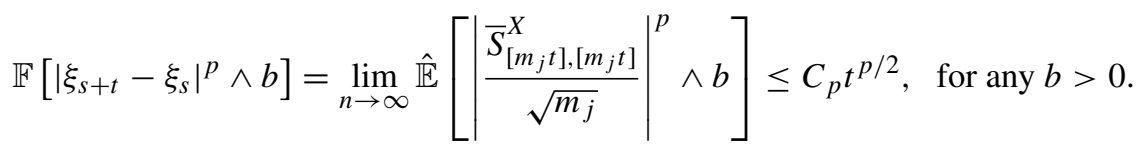

Hence,

$$
\mathbb{F}\left[\left|\xi_{s+t}-\xi_{s}\right|^{p}\right]=\lim _{b \rightarrow \infty} \mathbb{F}\left[\left|\xi_{s+t}-\xi_{s}\right|^{p} \wedge b\right] \leq C_{p} t^{p / 2}
$$

by the completeness of $\left(\bar{\Omega}, L_{\mathbb{F}}(\bar{\Omega}), \mathbb{F}\right)$. (29) is proved.

Now, note that $\left(X_{i}, Y_{i}\right), i=1,2, \ldots$, are independent and identically distributed. By (26) and Lemma 5, it is easily seen that $\left(\xi\right.$., $\eta$.) satisfies (14) for $\varphi \in C_{b}\left(\mathbb{R}^{2(k+1)}\right)$. Note that, by (29), the random variables concerned in (14) and (28) have finite 
moments of each order. The function space $C_{b}\left(\mathbb{R}^{2(k+1)}\right)$ and $C_{b}\left(\mathbb{R}^{2}\right)$ can be extended to $C_{l, L i p}\left(\mathbb{R}^{2(k+1)}\right)$ and $C_{l, L i p}\left(\mathbb{R}^{2}\right)$, respectively, by elemental arguments. So, (ii) and (iii) are proved.

For (iv) and (v), we let $\varphi: \mathbb{R}^{2} \rightarrow \mathbb{R}$ be a bounded Lipschitz function and consider

$$
u(x, y, t)=\mathbb{F}\left[\varphi\left(x+\xi_{t}, y+\eta_{t}\right)\right] .
$$

It is sufficient to show that $u$ is a viscosity solution of the PDE (13). In fact, due to the uniqueness of the viscosity solution, we will have

$$
\mathbb{F}\left[\varphi\left(x+\xi_{t}, y+\eta_{t}\right)\right]=\widetilde{\mathbb{E}}[\varphi(x+\sqrt{t} \xi, y+t \eta)], \varphi \in C_{b, L i p}\left(\mathbb{R}^{2}\right) .
$$

Letting $x=0$ and $y=0$ yields (iv) and (v).

To verify PDE (13), first it is easily seen that

$$
\hat{\mathbb{E}}\left[\frac{q}{2}\left(\frac{S_{[n t]}^{X}}{\sqrt{n}}\right)^{2}+p \frac{S_{[n t]}^{Y}}{n}\right]=\frac{[n t]}{n} \hat{\mathbb{E}}\left[\frac{q}{2}\left(\frac{S_{[n t]}^{X}}{\sqrt{[n t]}}\right)^{2}+p \frac{S_{[n t]}^{Y}}{[n t]}\right]=\frac{[n t]}{n} G(p, q) .
$$

Note that $\left\{\frac{q}{2}\left(\frac{S_{[n t]}^{X}}{\sqrt{n}}\right)^{2}+p \frac{S_{[n t]}^{Y}}{n}\right\}$ is uniformly integrable by Lemma 8. By Lemma 4, we conclude that

$$
\mathbb{F}\left[\frac{q}{2} \xi_{t}^{2}+p \eta_{t}\right]=\lim _{m_{j} \rightarrow \infty} \hat{\mathbb{E}}\left[\frac{q}{2}\left(\frac{S_{\left[m_{j} t\right]}^{X}}{\sqrt{m_{j}}}\right)^{2}+p \frac{S_{\left[m_{j} t\right]}^{Y}}{m_{j}}\right]=t G(p, q) .
$$

It is obvious that if $q_{1} \leq q_{2}$, then $G\left(p, q_{1}\right)-G\left(p, q_{2}\right) \leq G\left(0, q_{1}-q_{2}\right) \leq$ 0 . Also, it is easy to verify that $|u(x, y, t)-u(\bar{x}, \bar{y}, t)| \leq C(|x-\bar{x}|+|y-\bar{y}|)$, $|u(x, y, t)-u(x, y, s)| \leq C \sqrt{|t-s|}$ by the Lipschitz continuity of $\varphi$, and

$$
\begin{aligned}
u(x, y, t) & =\mathbb{F}\left[\varphi\left(x+\xi_{s}+\xi_{t}-\xi_{s}, y+\eta_{s}+\eta_{t}-\eta_{s}\right)\right] \\
& =\mathbb{F}\left[\left.\mathbb{F}\left[\varphi\left(x+\bar{x}+\xi_{t}-\xi_{s}, y+\bar{y}+\eta_{t}-\eta_{s}\right)\right]\right|_{(\bar{x}, \bar{y})=\left(\xi_{s}, \eta_{s}\right)}\right] \\
& =\mathbb{F}\left[u\left(x+\xi_{s}, y+\eta_{s}, t-s\right)\right], 0 \leq s \leq t .
\end{aligned}
$$

Let $\psi(\cdot, \cdot, \cdot) \in C_{b}^{3,3,2}(\mathbb{R}, \mathbb{R},[0,1])$ be a smooth function with $\psi \geq u$ and $\psi(x, y, t)=u(x, y, t)$. Then,

$$
\begin{aligned}
0 & =\mathbb{F}\left[u\left(x+\xi_{s}, y+\eta_{s}, t-s\right)-u(x, y, t)\right] \leq \mathbb{F}\left[\psi\left(x+\xi_{s}, y+\eta_{s}, t-s\right)-\psi(x, y, t)\right] \\
& =\mathbb{F}\left[\partial_{x} \psi(x, y, t) \xi_{s}+\frac{1}{2} \partial_{x x}^{2} \psi(x, y, t) \xi_{s}^{2}+\partial_{y} \psi(x, y, t) \eta_{s}-\partial_{t} \psi(x, y, t) s+I_{s}\right] \\
& \leq \mathbb{F}\left[\partial_{x} \psi(x, y, t) \xi_{s}+\frac{1}{2} \partial_{x x}^{2} \psi(x, y, t) \xi_{s}^{2}+\partial_{y} \psi(x, y, t) \eta_{s}-\partial_{t} \psi(x, y, t) s\right]+\mathbb{F}\left[\left|I_{s}\right|\right] \\
& =\mathbb{F}\left[\frac{1}{2} \partial_{x x}^{2} \psi(x, y, t) \xi_{s}^{2}+\partial_{y} \psi(x, y, t) \eta_{s}\right]-\partial_{t} \psi(x, y, t) s+\mathbb{F}\left[\left|I_{s}\right|\right] \\
& =s G\left(\partial_{y} \psi(x, y, t), \partial_{x x}^{2} \psi(x, y, t)\right)-s \partial_{t} \psi(x, y, t)+\mathbb{F}\left[\left|I_{s}\right|\right],
\end{aligned}
$$


where

$$
\left|I_{s}\right| \leq C\left(\left|\xi_{s}\right|^{3}+\left|\eta_{s}\right|^{2}+s^{2}\right) .
$$

By (29), we have $\mathbb{F}\left[\left|I_{s}\right|\right] \leq C\left(s^{3 / 2}+s^{2}+s^{2}\right)=o(s)$. It follows that $\left[\partial_{t} \psi-\right.$ $\left.G\left(\partial_{y} \psi, \partial_{x x}^{2}\right)\right](x, y, t) \leq 0$. Thus, $u$ is a viscosity subsolution of (13). Similarly, we can prove that $u$ is a viscosity supersolution of (13). Hence, (15) is proved.

As for (16), let $\varphi: C[0,1] \times C[0,1] \rightarrow \mathbb{R}$ be a continuous function with $|\varphi(x, y)| \leq C_{0}\left(1+\|x\|^{p}+\|y\|^{q}\right)$. For $\lambda>4 C_{0}$, let $\varphi_{\lambda}(x, y)=(-\lambda) \vee(\varphi(x, y) \wedge$ $\lambda) \in C_{b}(C[0,1])$. It is easily seen that $\varphi(x, y)=\varphi_{\lambda}(x, y)$ if $|\varphi(x, y)| \leq \lambda$. If $|\varphi(x, y)|>\lambda$, then

$$
\begin{gathered}
\left|\varphi(x, y)-\varphi_{\lambda}(x, y)\right|=|\varphi(x, y)|-\lambda \leq C_{0}\left(1+\|x\|^{p}+\|y\|^{q}\right)-\lambda \\
\leq C_{0}\left\{\left(\|x\|^{p}-\lambda /\left(4 C_{0}\right)\right)^{+}+\left(\|y\|^{q}-\lambda /\left(4 C_{0}\right)\right)^{+}\right\} .
\end{gathered}
$$

Hence,

$$
\left|\varphi(x, y)-\varphi_{\lambda}(x, y)\right| \leq C_{0}\left\{\left(\|x\|^{p}-\lambda /\left(4 C_{0}\right)\right)^{+}+\left(\|y\|^{q}-\lambda /\left(4 C_{0}\right)\right)^{+}\right\} .
$$

It follows that

$$
\begin{aligned}
& \lim _{\lambda \rightarrow \infty} \limsup _{n \rightarrow \infty}\left|\hat{\mathbb{E}}^{*}\left[\varphi\left(\widetilde{\boldsymbol{W}}_{n}(\cdot)\right)\right]-\hat{\mathbb{E}}\left[\varphi_{\lambda}\left(\widetilde{\boldsymbol{W}}_{n}(\cdot)\right)\right]\right| \\
\leq & \lim _{\lambda \rightarrow \infty} \limsup _{n \rightarrow \infty} C_{0}\left\{\hat{\mathbb{E}}\left[\left(\max _{k \leq n}\left|\frac{S_{k}^{X}}{\sqrt{n}}\right|^{p}-\frac{\lambda}{4 C_{0}}\right)^{+}\right]+\hat{\mathbb{E}}\left[\left(\max _{k \leq n}\left|\frac{S_{k}^{Y}}{n}\right|^{q}-\frac{\lambda}{4 C_{0}}\right)^{+}\right]\right\}
\end{aligned}
$$
$=0$,

by Lemma 8. Further, by (15),

$$
\lim _{n \rightarrow \infty} \hat{\mathbb{E}}\left[\varphi_{\lambda}\left(\widetilde{\boldsymbol{W}}_{n}(\cdot)\right)\right]=\widetilde{\mathbb{E}}\left[\varphi_{\lambda}(B ., b .)\right] \rightarrow \widetilde{\mathbb{E}}[\varphi(B ., b .)] \text { as } \lambda \rightarrow \infty .
$$

(16) is proved, and the proof of Theorem 4 is now completed.

Proof of Theorem 4. When $X_{k}$ and $Y_{k}$ are $d$-dimensional random vectors, the tightness (24) of $\widetilde{W}_{n}(\cdot)$ also follows, because each sequence of the components of vector $\widetilde{\boldsymbol{W}}_{n}(\cdot)$ is tight. Also, (29) remains true, because each component has this property. Moreover, it follows that

$$
\begin{aligned}
\mathbb{F}\left[\frac{1}{2}\left\langle A \xi_{t}, \xi_{t}\right\rangle+\left\langle p, \eta_{t}\right\rangle\right] & =\lim _{m_{j} \rightarrow \infty} \hat{\mathbb{E}}\left[\frac{1}{2}\left\langle A \frac{S_{\left[m_{j} t\right]}^{X}}{\sqrt{m_{j}}}, \frac{S_{\left[m_{j} t\right]}^{X}}{\sqrt{m_{j}}}\right\rangle+\left\langle p, \frac{S_{\left[m_{j} t\right]}^{Y}}{m_{j}}\right\rangle\right] \\
& =\lim _{m_{j} \rightarrow \infty} \frac{\left[m_{j} t\right]}{m_{j}} G(p, A)=t G(p, A) .
\end{aligned}
$$

The remaining proof is the same as that of Theorem 4 . 


\section{Proof of the self-normalized FCLTs}

Let $Y_{k}=X_{k}^{2}$. The function $G(p, q)$ in (12) becomes

$$
G(p, q)=\hat{\mathbb{E}}\left[\left(\frac{q}{2}+p\right) X_{1}^{2}\right]=\left(\frac{q}{2}+p\right)^{+} \bar{\sigma}^{2}-\left(\frac{q}{2}+p\right)^{-} \underline{\sigma}^{2}, \quad p, q \in \mathbb{R} .
$$

Then, the process $\left(B_{t}, b_{t}\right)$ in (15) and the process $\left(W(t),\langle W\rangle_{t}\right)$ are identically distributed.

In fact, note

$$
\langle W\rangle_{t+s}-\langle W\rangle_{t}=(W(t+s)-W(t))^{2}-2 \int_{0}^{s}(W(t+x)-W(t)) d(W(t+x)-W(t)) .
$$

It is easy to verify that $\left(W(t),\langle W\rangle_{t}\right)$ satisfies (i)-(iv) for $(B ., b$.). It remains to show that $\left(B_{1}, b_{1}\right) \stackrel{d}{=}\left(W(1),\langle W\rangle_{1}\right)$. Let $\left\{X_{n} ; n \geq 1\right\}$ be a sequence of independent and identically distributed random variables with $X_{1} \stackrel{d}{=} W(1)$. Then, by Theorem 4,

$$
\left(\frac{\sum_{k=1}^{n} X_{k}}{\sqrt{n}}, \frac{\sum_{k=1}^{n} X_{k}^{2}}{n}\right) \stackrel{d}{\rightarrow}\left(B_{1}, b_{1}\right)
$$

Further, let $t_{k}=\frac{k}{n}$. Then,

$$
\left(\frac{\sum_{k=1}^{n} X_{k}}{\sqrt{n}}, \frac{\sum_{k=1}^{n} X_{k}^{2}}{n}\right) \stackrel{d}{=}\left(W(1), \sum_{k=1}^{n}\left(W\left(t_{k}\right)-W\left(t_{k-1}\right)\right)^{2}\right) \stackrel{L_{2}}{\rightarrow}\left(W(1),\langle W\rangle_{1}\right) .
$$

Hence, $(B ., b.) \stackrel{d}{=}(W(\cdot),\langle W\rangle$.). We conclude the following proposition from Theorem 4.

Proposition 1 Suppose $\hat{\mathbb{E}}\left[\left(X_{1}^{2}-b\right)^{+}\right] \rightarrow 0$ as $b \rightarrow \infty$. Then, for any bounded continuous function $\psi: C[0,1] \times C[0,1] \rightarrow \mathbb{R}$,

$$
\hat{\mathbb{E}}\left[\psi\left(\frac{\widetilde{S}_{n}^{X}(\cdot)}{\sqrt{n}}, \frac{\widetilde{V}_{n}(\cdot)}{n}\right)\right] \rightarrow \widetilde{\mathbb{E}}[\psi(W(\cdot),\langle W\rangle .)],
$$

where $\widetilde{V}_{n}(t)=V_{[n t]}+(n t-[n t]) X_{[n t]+1}^{2}$, and, in particular, for any bounded continuous function $\psi: C[0,1] \times \mathbb{R} \rightarrow \mathbb{R}$,

$$
\hat{\mathbb{E}}\left[\psi\left(\frac{\widetilde{S}_{n}^{X}(\cdot)}{\sqrt{n}}, \frac{V_{n}}{n}\right)\right] \rightarrow \widetilde{\mathbb{E}}\left[\psi\left(W(\cdot),\langle W\rangle_{1}\right)\right] .
$$

Now, we begin the proof of Theorem 2. Let $a=\sigma^{2} / 2$ and $b=2 \bar{\sigma}^{2}$. According to (30), we have $\mathcal{V}\left(\underline{\sigma}^{2}-\epsilon<\langle W\rangle_{1}<\bar{\sigma}^{2}+\epsilon\right)=1$ for all $\epsilon>0$. Let $\varphi: C[0,1] \rightarrow \mathbb{R}$ be a bounded continuous function. Define

$$
\psi(x(\cdot), y)=\varphi\left(\frac{x(\cdot)}{\sqrt{a \vee y \wedge b}}\right), \quad x(\cdot) \in C[0,1], y \in \mathbb{R} .
$$


Then, $\psi: C[0,1] \times \mathbb{R} \rightarrow \mathbb{R}$ is a bounded continuous function. Hence, by Proposition 1,

$\hat{\mathbb{E}}\left[\varphi\left(\frac{\widetilde{S}_{n}^{X}(\cdot) / \sqrt{n}}{\sqrt{a \vee\left(V_{n} / n\right) \wedge b}}\right)\right] \rightarrow \widetilde{\mathbb{E}}\left[\varphi\left(\frac{W(\cdot)}{\sqrt{a \vee\left(\langle W\rangle_{1}\right) \wedge b}}\right)\right]=\widetilde{\mathbb{E}}\left[\varphi\left(\frac{W(\cdot)}{\sqrt{\left.\langle W\rangle_{1}\right)}}\right)\right]$.

Also,

$$
\begin{aligned}
\limsup _{n \rightarrow \infty} & \left|\hat{\mathbb{E}}^{*}\left[\varphi\left(\frac{\widetilde{S}_{n}^{X}(\cdot) / \sqrt{n}}{\sqrt{V_{n} / n}}\right)\right]-\hat{\mathbb{E}}\left[\varphi\left(\frac{\widetilde{S}_{n}^{X}(\cdot) / \sqrt{n}}{\sqrt{a \vee\left(V_{n} / n\right) \wedge b}}\right)\right]\right| \\
& \leq C \limsup _{n \rightarrow \infty} \mathbb{V}\left(V_{n} / n \notin(a, b)\right) \\
& \leq C \widetilde{\mathbb{V}}\left(\langle W\rangle_{1} \geq 3 \bar{\sigma}^{2} / 2\right)+C \widetilde{\mathbb{V}}\left(\langle W\rangle_{1} \leq 2 \underline{\sigma}^{2} / 3\right)=0 .
\end{aligned}
$$

It follows that

$$
\hat{\mathbb{E}}^{*}\left[\varphi\left(\frac{\widetilde{S}_{n}^{X}(\cdot)}{\sqrt{V_{n}}}\right)\right] \rightarrow \widetilde{\mathbb{E}}\left[\varphi\left(\frac{W(\cdot)}{\sqrt{\left.\langle W\rangle_{1}\right)}}\right)\right] .
$$

The proof is now completed.

Proof of Theorem 3. First, note that

$$
\begin{aligned}
\hat{\mathbb{E}}\left[X_{1}^{2} \wedge x^{2}\right] & \leq \hat{\mathbb{E}}\left[X_{1}^{2} \wedge(k x)^{2}\right] \leq \hat{\mathbb{E}}\left[X_{1}^{2} \wedge x^{2}\right]+k^{2} x^{2} \mathbb{V}\left(\left|X_{1}\right|>x\right), \quad k \geq 1, \\
\hat{\mathbb{E}}\left[\left|X_{1}\right|^{r} \wedge x^{r}\right] & \leq \hat{\mathbb{E}}\left[\left|X_{1}\right|^{r} \wedge(\delta x)^{r}\right]+\hat{\mathbb{E}}\left[(\delta x)^{r} \vee\left|X_{1}\right|^{r} \wedge x^{r}\right] \\
& \leq \delta^{r-2} x^{r-2} l(\delta x)+x^{r} \mathbb{V}\left(\left|X_{1}\right| \geq \delta x\right), \quad 0<\delta<1, r>2 .
\end{aligned}
$$

The condition (I) implies that $l(x)$ is slowly varying as $x \rightarrow \infty$ and

$$
\hat{\mathbb{E}}\left[\left|X_{1}\right|^{r} \wedge x^{r}\right]=o\left(x^{r-2} l(x)\right), r>2 .
$$

Further,

$$
\begin{gathered}
\frac{\hat{\mathbb{E}}^{*}\left[X_{1}^{2} I\left\{\left|X_{1}\right| \leq x\right\}\right]}{l(x)} \rightarrow 1, \\
C_{\mathbb{V}}\left(\left|X_{1}\right|^{r} I\left\{\left|X_{1}\right| \geq x\right\}\right)=\int_{x^{r}}^{\infty} \mathbb{V}\left(\left|X_{1}\right|^{r} \geq y\right) d y=o\left(x^{2-r} l(x)\right), \quad 0<r<2 .
\end{gathered}
$$

If conditions (I) and (III) are satisfied, then

$$
\hat{\mathbb{E}}\left[\left(\left|X_{1}\right|-x\right)^{+}\right] \leq \hat{\mathbb{E}}^{*}\left[\left|X_{1}\right| I\{|X| \geq x\}\right] \leq C_{\mathbb{V}}\left(\left|X_{1}\right| I\left\{\left|X_{1}\right| \geq x\right\}\right)=o\left(x^{-1} l(x)\right) .
$$

Now, let $d_{t}=\inf \left\{x: x^{-2} l(x)=t^{-1}\right\}$. Then, $n l\left(d_{n}\right)=d_{n}^{2}$. Similar to Theorem 2, it is sufficient to show that for any bounded continuous function $\psi: C[0,1] \times$ $C[0,1] \rightarrow \mathbb{R}$, 
$\hat{\mathbb{E}}\left[\psi\left(\frac{\widetilde{S}_{n}^{X}(\cdot)}{d_{n}}, \frac{\widetilde{V}_{n}(\cdot)}{d_{n}^{2}}\right)\right] \rightarrow \widetilde{\mathbb{E}}[\psi(W(\cdot),\langle W\rangle)$.$] with W(1) \sim N\left(0,\left[r^{-2}, 1\right]\right)$.

Let $\bar{X}_{k}=\bar{X}_{k, n}=\left(-d_{n}\right) \vee X_{k} \wedge d_{n}, \bar{S}_{k}=\sum_{i=1}^{k} \bar{X}_{i}, \bar{V}_{k}=\sum_{i=1}^{k} \bar{X}_{i}^{2}$. Denote $\bar{S}_{n}(t)=\bar{S}_{[n t]}+(n t-[n t]) \bar{X}_{[n t]+1}$ and $\bar{V}_{n}(t)=\bar{V}_{[n t]}+(n t-[n t]) \bar{X}_{[n t]+1}^{2}$. Note

$$
\mathbb{V}\left(X_{k} \neq \bar{X}_{k} \text { for some } k \leq n\right) \leq n \mathbb{V}\left(\left|X_{1}\right| \geq d_{n}\right)=n \cdot o\left(\frac{l\left(d_{n}\right)}{d_{n}^{2}}\right)=o(1) .
$$

It is sufficient to show that for any bounded continuous function $\psi: C[0,1] \times$ $C[0,1] \rightarrow \mathbb{R}$

$$
\hat{\mathbb{E}}\left[\psi\left(\frac{\bar{S}_{n}(\cdot)}{d_{n}}, \frac{\bar{V}_{n}(\cdot)}{d_{n}^{2}}\right)\right] \rightarrow \widetilde{\mathbb{E}}[\psi(W(\cdot),\langle W\rangle .)] .
$$

Following the line of the proof of Theorem 4, we need only to show that

(a) for any $0<t \leq 1$,

$$
\limsup _{n \rightarrow \infty} \hat{\mathbb{E}}\left[\max _{k \leq[n t]}\left|\frac{\bar{S}_{k}}{d_{n}}\right|^{p}\right] \leq C_{p} t^{p / 2}, \quad \limsup _{n \rightarrow \infty} \hat{\mathbb{E}}\left[\max _{k \leq[n t]}\left|\frac{\bar{V}_{k}}{d_{n}^{2}}\right|^{p}\right] \leq C_{p} t^{p}, \quad \forall p \geq 2 ;
$$

(b) for any $0<t \leq 1$,

$$
\lim _{n \rightarrow \infty} \hat{\mathbb{E}}\left[\frac{q}{2}\left(\frac{\bar{S}_{[n t]}}{d_{n}}\right)^{2}+p \frac{\bar{V}_{[n t]}}{d_{n}^{2}}\right]=t G(p, q)
$$

where

$$
G(p, q)=\left(\frac{q}{2}+p\right)^{+}-r^{-2}\left(\frac{q}{2}+p\right)^{-}
$$

(c)

$$
\max _{k \leq n} \frac{\left|X_{k}\right|}{d_{n}} \stackrel{\mathbb{V}}{\rightarrow} 0
$$

In fact, (a) implies the tightness of $\left(\frac{\widetilde{S}_{n}^{X}(\cdot)}{d_{n}}, \frac{\widetilde{V}_{n}(\cdot)}{d_{n}^{2}}\right)$ and (29), and (b) implies the distribution of the limit process is uniquely determined.

First, (c) is obvious, because

$$
\mathbb{V}\left(\max _{k \leq n}\left|X_{k}\right| \geq \epsilon d_{n}\right) \leq n \mathbb{V}\left(\left|X_{1}\right| \geq \epsilon d_{n}\right)=o(1) n \frac{l\left(\epsilon d_{n}\right)}{\epsilon^{2} d_{n}^{2}}=o(1) n \frac{l\left(d_{n}\right)}{d_{n}^{2}}=o(1)
$$


As for (a), by the Rosenthal-type inequality (18),

$$
\begin{aligned}
\hat{\mathbb{E}}\left[\max _{k \leq[n t]}\left|\frac{\bar{S}_{k}}{d_{n}}\right|^{p}\right] \leq C_{p} d_{n}^{-p}\left\{[n t] \hat{\mathbb{E}}\left[\left|X_{1}\right|^{p} \wedge d_{n}^{p}\right]+\left([n t] \hat{\mathbb{E}}\left[\left|X_{1}\right|^{2} \wedge d_{n}^{2}\right]\right)^{p / 2}\right. \\
\left.\quad+\left([n t]\left(\widehat{\mathcal{E}}\left[\left(-d_{n}\right) \vee X_{1} \wedge d_{n}\right]\right)^{+}+[n t]\left(\hat{\mathbb{E}}\left[\left(-d_{n}\right) \vee X_{1} \wedge d_{n}\right]\right)^{+}\right)^{p}\right\} \\
\quad \leq C_{p} d_{n}^{-p}\left\{[n t] \hat{\mathbb{E}}\left[\left|X_{1}\right|^{p} \wedge d_{n}^{p}\right]+\left([n t] \hat{\mathbb{E}}\left[\left|X_{1}\right|^{2} \wedge d_{n}^{2}\right]\right)^{p / 2}+\left([n t] \hat{\mathbb{E}}\left[\left(\left|X_{1}\right|-d_{n}\right)^{+}\right]\right)^{p}\right\} \\
\quad \leq C_{p} d_{n}^{-p}\left\{[n t] o\left(d_{n}^{p-2} l\left(d_{n}\right)\right)+\left([n t] l\left(d_{n}\right)\right)^{p / 2}+\left([n t] o\left(\frac{l\left(d_{n}\right)}{d_{n}}\right)\right)^{p}\right\} \\
\quad=o(1)[n t] \frac{l\left(d_{n}\right)}{d_{n}^{2}}+\left(\frac{[n t]}{n}\right)^{p / 2}\left(\frac{n l\left(d_{n}\right)}{d_{n}^{2}}\right)^{p / 2}+o(1)\left([n t] \frac{l\left(d_{n}\right)}{d_{n}^{2}}\right)^{p} \leq C_{p} t^{p / 2}+o(1),
\end{aligned}
$$

and similarly,

$$
\begin{aligned}
\hat{\mathbb{E}}\left[\max _{k \leq[n t]}\left|\frac{\bar{V}_{k}}{d_{n}^{2}}\right|^{p}\right] \leq & C_{p} d_{n}^{-2 p}\left\{[n t] \hat{\mathbb{E}}\left[\left|X_{1}\right|^{2 p} \wedge d_{n}^{2 p}\right]+\left([n t] \hat{\mathbb{E}}\left[\left|X_{1}\right|^{4} \wedge d_{n}^{4}\right]\right)^{p / 2}\right. \\
& \left.+\left([n t] \widehat{\mathcal{E}}\left[X_{1}^{2} \wedge d_{n}^{2}\right]\right)+[n t]\left(\hat{\mathbb{E}}\left[X_{1}^{2} \wedge d_{n}^{2}\right]\right)^{p}\right\} \\
= & o(1)+C_{p}\left([n t] \frac{l\left(d_{n}\right)}{d_{n}^{2}}\right)^{p} \leq C_{p} t^{p}+o(1) .
\end{aligned}
$$

Thus (a) follows.

As for (b), note

$$
\frac{q}{2}\left(\frac{\bar{S}_{[n t]}}{d_{n}}\right)^{2}+p \frac{\bar{V}_{[n t]}}{d_{n}^{2}}=\left(\frac{q}{2}+p\right) \frac{\bar{V}_{[n t]}}{d_{n}^{2}}+q \frac{\sum_{k=1}^{[n t]-1} \bar{S}_{k-1} \bar{X}_{k}}{d_{n}^{2}} .
$$

By (32),

$$
\begin{aligned}
\hat{\mathbb{E}}\left[\sum_{k=1}^{[n t]-1} \bar{S}_{k-1} \bar{X}_{k}\right] & \leq \sum_{k=1}^{[n t]-1} \hat{\mathbb{E}}\left[\bar{S}_{k-1} \bar{X}_{k}\right] \\
& \leq \sum_{k=1}^{[n t]-1}\left\{\hat{\mathbb{E}}\left[\left(\bar{S}_{k-1}\right)^{+}\right] \hat{\mathbb{E}}\left[\bar{X}_{k}\right]-\hat{\mathbb{E}}\left[\left(\bar{S}_{k-1}\right)^{-}\right] \widehat{\mathcal{E}}\left[\bar{X}_{k}\right]\right\} \\
& \leq \sum_{k=1}^{[n t]-1}\left(\hat{\mathbb{E}}\left[\left|\bar{S}_{k-1}\right|^{2}\right]\right)^{1 / 2} \hat{\mathbb{E}}\left[\left(\left|X_{1}\right|-d_{n}\right)^{+}\right] \\
& =O\left(\left(d_{n}^{2}\right)^{1 / 2}\right) \cdot n \hat{\mathbb{E}}\left[\left(\left|X_{1}\right|-d_{n}\right)^{+}\right] \\
& =O\left(d_{n}\right) \cdot n \cdot o\left(\frac{l\left(d_{n}\right)}{d_{n}}\right)=o\left(d_{n}^{2}\right),
\end{aligned}
$$

and similarly, 


$$
\hat{\mathbb{E}}\left[-\sum_{k=1}^{[n t]-1} \bar{S}_{k-1} \bar{X}_{k}\right]=o\left(d_{n}^{2}\right)
$$

Further,

$$
\frac{\hat{\mathbb{E}}\left[V_{[n t]}\right]}{d_{n}^{2}}=\frac{[n t] \hat{\mathbb{E}}\left[X_{1}^{2} \wedge d_{n}^{2}\right]}{d_{n}^{2}}=\frac{[n t]}{n} \frac{n l\left(d_{n}\right)}{d_{n}^{2}}=\frac{[n t]}{n} \rightarrow t
$$

and

$$
\frac{\widehat{\mathcal{E}}\left[V_{[n t]}\right]}{d_{n}^{2}}=\frac{[n t] \widehat{\mathcal{E}}\left[X_{1}^{2} \wedge d_{n}^{2}\right]}{d_{n}^{2}}=\frac{[n t]]}{n} \frac{\widehat{\mathcal{E}}\left[X_{1}^{2} \wedge d_{n}^{2}\right]}{\hat{\mathbb{E}}\left[X_{1}^{2} \wedge d_{n}^{2}\right]} \rightarrow t r^{-2}
$$

Hence, we conclude that

$$
\begin{gathered}
\hat{\mathbb{E}}\left[\frac{q}{2}\left(\frac{\bar{S}_{[n t]}}{d_{n}}\right)^{2}+p \frac{\bar{V}_{[n t]}}{d_{n}^{2}}\right]=\hat{\mathbb{E}}\left[\left(\frac{q}{2}+p\right) \frac{\bar{V}_{[n t]}}{d_{n}^{2}}\right]+o(1) \\
=t\left[\left(\frac{q}{2}+p\right)^{+}-r^{-2}\left(\frac{q}{2}+p\right)^{-}\right]+o(1) .
\end{gathered}
$$

Thus, (b) is statisfied, and the proof is completed.

Acknowledgements Research supported by Grants from the National Natural Science Foundation of China (No. 11225104), the 973 Program (No. 2015CB352302) and the Fundamental Research Funds for the Central Universities.

\section{Authors' contributions}

All authors have equal contributions to the paper. All authors read and approved the final manuscript.

\section{Competing interests}

The authors declare that they have no competing interests.

\section{References}

Csörgő, M, Szyszkowicz, B, Wang, QY: Donsker's theorem for self-normalized partial sums processes. Ann. Probab 31, 1228-1240 (2003)

Denis, L, Hu, MS, Peng, SG: Function spaces and capacity related to a sublinear expectation: application to G-Brownian Motion Paths. Potential Anal 34, 139-161 (2011). arXiv:0802.1240v1 [math.PR]

Giné, E, Götze, F, Mason, DM: When is the Student $t$-statistic asymptotically standard normal? Ann. Probab 25, 1514-1531 (1997)

Hu, MS, Ji, SL, Peng, SG, Song, YS: Backward stochastic differential equations driven by G-Brownian motion. Stochastic Process. Appl 124(1), 759-784 (2014a)

Hu, MS, Ji, SL, Peng, SG, Song, YS: Comparison theorem, Feynman-Kac formula and Girsanov transformation for BSDEs driven by G-Brownian motion. Stochastic Process. Appl 124(2), 1170-1195 (2014b)

Li, XP, Peng, SG: Topping times and related Ito's calculus with G-Brownian motion. Stochastic Process. Appl 121(7), 1492-1508 (2011) 
Nutz, M, van Handel, R: Constructing sublinear expectations on path space. Stochastic Process. Appl 123(8), 3100-3121 (2013)

Peng, SG: G-expectation, G-Brownian motion and related stochastic calculus of Ito's type. The Abel Symposium 2005, Abel Symposia 2, Edit. Benth et. al, pp. 541-567. Springer-Verlag (2006)

Peng, SG: Multi-dimensional G-Brownian motion and related stochastic calculus under G-expectation. Stochastic Process. Appl 118(12), 2223-2253 (2008a)

Peng, SG: A new central limit theorem under sublinear expectations (2008b). Preprint: arXiv:0803.2656v1 [math.PR]

Peng, SG: Survey on normal distributions, central limit theorem, Brownian motion and the related stochastic calculus under sublinear expectations. Sci. China Ser. A 52(7), 1391-1411 (2009)

Peng, SG: Nonlinear Expectations and Stochastic Calculus under Uncertainty (2010a). Preprint: arXiv: 1002.4546 [math.PR]

Peng, SG: Tightness, weak compactness of nonlinear expectations and application to CLT (2010b). Preprint: arXiv:1006.2541 [math.PR]

Yan, D, Hutz, M, Soner, HM: Weak approximation of G-expectations. Stochastic Process. Appl 122(2), 664-675 (2012)

Zhang, LX: Donsker's invariance principle under the sub-linear expectation with an application to Chung's law of the iterated logarithm. Commun. Math. Stat 3(2), 187-214 (2015). arXiv:1503.02845 [math.PR]

Zhang, LX: Rosenthal's inequalities for independent and negatively dependent random variables under sub-linear expectations with applications. Sci. China Math 59(4), 751-768 (2016) 